\title{
ÜBER EINE VERALLGEMEINERUNG DER FOURIERSCHEN INTEGRALFORMEL.
}

\author{
VoN \\ HANS HAHN \\ in WIEN.
}

Im Folgenden soll das Fouriersche Integraltheorem in einer Gestalt bewiesen werden, die infolge Heranziehung von Stieltjesintegralen den Geltungsbereich dieses Theorems beträchtlich erweitert. Fs fallen z. B. in ihren Geltungsbereich alle Funktionen, die an der betrachteten Stelle $x_{0}$ in die Fourierreihe entwickelbar sind, und im Unendlichen eine der folgenden Bedingungen erfüllen: I) es ist $\left|\frac{f(x)}{x}\right|$ im Unendlichen integrierbar; 2) $f(x)$ ist das Produkt einer periodischen Funktion und einer Funktion, die im Unendlichen beschränkt und monoton ist; 3) $[f(x)]^{2}$ ist im Unendlichen integrierbar. ${ }^{1}$ Zum Falle 2) gehören insbesondere alle an der Stelle $x_{0}$ in die Fourierreihe entwickelbaren periodischen Funktionen, und für diese reduziert sich unsere Formel einfach auf die Fourierreihe, die so als Stieltjessches Fourierintegral erscheint. In den Fällen, in denen $f(x)$ durch die klassische Fouriersche Integralformel darstellbar ist, reduziert sich unsere Formel auf diese, und gestattet so zugleich, nicht nur die wichtigsten Bedingungen für die Giltigkeit der klassischen Fourierschen Integralformel ${ }^{2}$ einfach und durchsichtig zu begründen, sondern auch neue, sehr allgemeine Bedingungen für die Giltigkeit dieser Formel aufzustellen. An andrer Stelle hoffe

1 Dieser Teil unsrer Untersuchungen berührt sich enge mit Untersuchungen von M. PLANCHERer, Rend. Pal. 30 (I9IO), S. 289, Math. Ann. 74 (I9I3), S. 573; Math. Ann. 76 (I915), S. 315.

2 Etwa die von A. Pringsfirm angegebenen: Math. Ann. 68 (I9Io), S. 367 ; Math. Ann. 7 I (I9I I), S. 289 . 
ich, bald zeigen zu können, wie sich die hier vorgebrachten Untersuchungen auf alle Funktionen ausdehnen lassen, die im Unendlichen beschränkt sind.

Die vorliegende Arbeit ist (mit Ausnahme des später hinzugefügten $§$ I9) die ausführliche Wiedergabe eines auf der lnnsbrucker Versammlung (September 1924) gehaltenen Vortrages, von dem eine kurze Inhaltsangabe bereits in den Jahresberichten der Deutschen Mathematikervereinigung erschienen ist. Zwei kurzen Noten ${ }^{1}$ entnehme ich, dass sich Herr N. WIEnkr mit demselben Probleme befasst hat. Ob seine Methoden mit den meinen in irgend einer Beziehung stehen, entzieht sich meiner Kenntnis.

\section{$\S \mathrm{I}$.}

Sei $f(x)$ eine in jedem endlichen Intervalle integrierbare ${ }^{2}$ Funktion, die obendrein folgende Figenschaften besitze:

A) An der Stelle $x_{0}$ sei $f(x)$ in die Fouriersche Reihe entwickelbar (d. h. für jede mit $f(x)$ in einer Umgebung von $x_{0}$ übereinstimmende Funktion der Periode $2 \pi$ konvergiere die Fouriersche Reihe an der Stelle $x_{0}$ und stelle den Funktionswert $f\left(x_{0}\right)$ dar).

B) Es sei $\frac{f(x)}{x}$ im Unendlichen absolut integrierbar (d. h. für $a>0$ fallen die beiden Grenzwerte $\lim _{q \rightarrow+\infty} \int_{a}^{q}\left|\begin{array}{c}f^{\prime}(x) \\ x\end{array}\right| d x$ und $\lim _{q \rightarrow+\infty} \int_{q}\left|\frac{f(x)}{x}\right| d x$ endlich aus). Unter diesen Voraussetzungen gilt die Formel:

$$
f\left(x_{0}\right)=\frac{1}{\pi} \lim _{\lambda \rightarrow+\infty} \int_{-\infty}^{+\infty} f(x) \frac{\sin \lambda\left(x-x_{0}\right)}{x-x_{0}} d x
$$

In der Tat, zunächst ist wegen Eigenschaft B), da ausserhalb des Inter-

1 Am. Bull. 3i (1925), S. 106, 22r. (Zusatz bei der Korrektur: die sehr bedeutungsvollen Lntersuchungen des Herrn WiENEK sind mittlerweile ausführlich erschienen : Math. Zeitschr. 24, S. 575).

${ }^{2}$ Integrierbarkeit ist im Folgenden stets im Sinne von LEBssauk zu verstehen; die anftretenden Integrale sind, wo nicht ausdrücklich das Gegenteil gesagt ist, Isebesguesche Integrale. Dabei bedeutet in üblicher Weise. $\int_{-\infty}^{+\infty}$ so viel wie $\lim _{p \rightarrow-\infty, q \rightarrow+\infty} \int_{p}^{q}$. Von allen auftretenden Funktionen wird vorausgesetzt, dass sie in jedem endlicher Intervalle integrierbar sind. 
valles $\left(x_{0}-\mathrm{I}, x_{0}+\mathrm{I}\right)$ der Ausdruck $\frac{x}{x-x_{0}} \sin \lambda\left(x-x_{0}\right)$ beschränkt ist, das in (I) auftretende Integral sicher vorhanden; ist ferner $\varepsilon>0$ beliebig gegeben, so kann $b>0$ so gewählt werden, dass für alle $\lambda$ :

(2) $\quad\left|\frac{\mathrm{I}}{\pi} \int_{x_{0}+b}^{+\infty} f(x) \frac{\sin \lambda\left(x-x_{0}\right)}{x-x_{0}} d x\right|<\varepsilon ;\left|\frac{\mathrm{I}}{\pi} \int_{-\infty}^{x_{0}-b} f(x) \frac{\sin \lambda\left(x-x_{0}\right)}{x-x_{0}} d x\right|<\varepsilon$.

Wegen Eigenschaft A) aber ist:

$$
\frac{\mathrm{I}}{\pi} \lim _{\lambda \rightarrow+\infty} \int_{x_{0}-b}^{x_{0}+b} f(x) \frac{\sin \lambda\left(x-x_{0}\right)}{x-x_{0}} d x=f\left(x_{0}\right)
$$

d. h. es gibt ein $\lambda_{0}$, so dass:

$$
\left|\frac{\mathrm{I}}{\pi} \int_{x_{0}-b}^{x_{0}+b} f(x) \frac{\sin \lambda\left(x-x_{0}\right)}{x-x_{0}} d x-f\left(x_{0}\right)\right|<\varepsilon \text { für } \lambda>\lambda_{0}
$$

Aus (2) und (3) folgt:

$$
\left|\frac{\mathrm{I}}{\pi} \int_{-\infty}^{+\infty} f(x) \frac{\sin \lambda\left(x-x_{0}\right)}{x-x_{0}} d x-f\left(x_{0}\right)\right|<3 \varepsilon \text { für } \lambda>\lambda_{0}
$$

womit (I) bewiesen ist.

Bezeichnet $n$ eine natürliche Zahl, so ist:

(4) $\int_{-\infty}^{+\infty} f(x) \frac{\sin \lambda\left(x-x_{0}\right)}{x-x_{0}} d x=\int_{-\infty}^{+\infty}\left(f(x) \int_{0}^{\lambda} \cos \mu\left(x-x_{0}\right) d \mu\right) d x$

Hierin nun ist:

$$
=\lim _{n \rightarrow \infty} \int_{-n}^{n}\left(f(x) \int_{0}^{\lambda} \cos \mu\left(x-x_{0}\right) d \mu\right) d x .
$$


(5) $\int_{-n}^{n}\left(f(x) \int_{0}^{\lambda} \cos \mu\left(x-x_{0}\right) d \mu\right) d x=\int_{0}^{\lambda}\left(\cos \mu x_{0} \int_{-n}^{n} f(x) \cos \mu x d x\right) d \mu+$

Setzen wir:

$$
+\int_{0}^{\lambda}\left(\sin \mu x_{0} \int_{-n}^{n} f(x) \sin \mu x d x\right) d \mu
$$

$$
\Phi_{n}(\mu)=\int_{-n}^{n} f(x) \frac{\sin \underline{\mu x}}{x} d x ; \Psi_{n}(\mu)=\int_{-n}^{n} f(x) \frac{\mathrm{I}-\cos \mu x}{x} d x
$$

so sind $\Phi_{n}(\mu)$ und $\Psi_{n}(\mu)$ stetige, differenzierbare Funktionen, und es ist:

$$
\frac{d \Phi_{n}(\mu)}{d \mu}=\int_{-n}^{n} f(x) \cos \mu x d x ; \quad \frac{d \Psi_{n}(\mu)}{d \mu}=\int_{-n}^{n} f(x) \sin \mu x d x
$$

so dass (5) auch geschrieben werden $\operatorname{kann}^{1}$ :

(6) $\int_{-n}^{n}\left(f(x) \int_{0}^{\lambda} \cos \mu\left(x-x_{0}\right) d \mu\right) d x=\int_{0}^{\lambda} \cos \mu x_{0} d \Phi_{n}(\mu)+\int_{0}^{\lambda} \sin \mu x_{0} d \Psi_{n}(\mu)$.

Hierin nun ist der Grenzübergang $n \rightarrow \infty$ zu vollziehen.

Aus Eigenschaft B) folgt die Existenz der Integrale:

$$
\Phi(\mu)=\int_{-\infty}^{+\infty} f(x) \frac{\sin \mu x}{x} d x ; \quad \Psi(\mu)=\int_{-\infty}^{+\infty} f(x) \frac{\mathrm{I}-\cos \mu x}{x} d x
$$

und es ist:

$$
\lim _{n \rightarrow \infty} \Phi_{n}(\mu)=\Phi(\mu) ; \quad \lim _{n \rightarrow \infty} \Psi_{n}(\mu)=\Psi(\mu)
$$

Die Konvergenz hierin ist gleichmässig für alle $\mu$ des Intervalles $[0, \lambda]$; denn ist $\varepsilon>0$ beliebig gegeben, so kann man wegen Eigenschaft B) die Zahl $b$ so gross wählen, dass:

1 Über den hiebei zur Verwendung kommenden Integralbegriff vgl. Monatsh. f. Math. u. Phys. 32 (I922), S. 69 ff. 


$$
\int_{b}^{+\infty}\left|\frac{f(x)}{x}\right| d x<\varepsilon ; \quad \int_{-\infty}^{-h}\left|\frac{f(x)}{x}\right| d x<\varepsilon
$$

dann aber ist für alle $n>b$ und alle $\mu$ :

$$
\begin{aligned}
& \left|\Phi_{n}(\mu)-\Phi(\mu)\right|=\left|\int_{-\infty}^{\sigma^{n}} f(x) \frac{\sin \mu x}{x} d x+\int_{n}^{+\infty} f(x) \frac{\sin \mu x}{x} d x\right|<2 \varepsilon, \\
& \left|\Psi_{n}(\mu)-\Psi(\mu)\right|=\left|\int_{-\infty}^{-n} f(x) \frac{I-\cos \mu x}{x} d x+\int_{n}^{+\infty} f(x) \frac{1-\cos \mu x}{x} d x\right|<4 \varepsilon .
\end{aligned}
$$

Aus der Stetigkeit von $\Phi_{n}(\mu)$ und $\Psi_{n}(\mu)$ folgt also die Stetigkeit von $\boldsymbol{\Phi}(\mu)$ und $\Psi(\mu)$, und wir sehen ferner, dass die Gesamtheit der $\boldsymbol{\Phi}_{n}(\mu)$ und $\Psi_{n}(\mu)$ im Intervalle $[o, \lambda]$ zwischen endlichen Schranken bleibt:

(9)

$$
\left|\Phi_{n}(\mu)\right| \leqq M, \quad\left|\Psi_{n}(\mu)\right| \leqq M \text { für o } \leqq \mu \leqq \lambda \text { und alle } n \text {. }
$$

Aus (8) und (9) aber folgt. :

$$
\begin{aligned}
& \lim _{n \rightarrow \infty} \int_{0}^{\lambda} \cos \mu x_{0} d \Phi_{n}(\mu)=\int_{0}^{\lambda} \cos \mu x_{0} d \Phi(\mu), \\
& \lim _{n \rightarrow \infty} \int_{0}^{\lambda} \sin \mu x_{0} d \Psi_{n}(\mu)=\int_{0}^{\lambda} \sin \mu x_{0} d \Psi(\mu)
\end{aligned}
$$

Aus (1), (4) und (6) folgt somit:

$$
f\left(x_{0}\right)=\frac{I}{\pi} \lim _{\lambda \rightarrow+\infty}\left(\int_{0}^{\lambda} \cos \mu x_{0} d \Phi(\mu)+\int_{0}^{\lambda} \sin \mu x_{0} d \Psi(\mu)\right)
$$

wofür wir auch schreiben:

$$
f\left(x_{0}\right)=\frac{\mathrm{I}}{\pi} \int_{0}^{+\infty}\left(\cos \mu x_{0} d \Phi(\mu)+\sin \mu x_{0} d \Psi(\mu)\right)
$$

\footnotetext{
1. e. S. 304, S. $77-80$.
}

39-2661. Acta mathematica. 49. Imprimé le 29 juillet 1926. 
Wir haben also den Satz ${ }^{1}$

Satz I. Hat $f(x)$ die Eigenschaften A) und B), so gitt (10), worin $\Phi(\mu)$ und $\Psi(\mu)$ die Bedeutung (7) haben und stetige Funktionen von $\mu$ sind.

\section{$\$ 2$.}

Formel (1o) steht in naher Beziehung zur Fouvierschen Integralformel:

$$
f\left(x_{0}\right)=\frac{1}{\pi} \int_{0}^{+\infty}\left(\varphi(\mu) \cos \mu x_{0}+\psi(\mu) \sin \mu x_{0}\right) d \mu
$$

worin gesetzt ist:

$$
\varphi(\mu)=\int_{-\infty}^{+\infty} f(x) \cos \mu x d x ; \quad \psi(\mu)=\int_{-\infty}^{+\infty} f(x) \sin \mu x d x
$$

Doch ist die Tragweite der Formel (10) eine bei weitem grössere. Wir wollen zunächst an einem Beispiele zeigen, dass die für die Giltigkeit von (Io) hinreichenden Bedingungen A) und B) für die Giltigkeit der Fourierschen Integralformel (I I) keineswegs ausreichen. ${ }^{2}$

Die Funktion $f(x)$ sei gerade, sie sei konstant in jedem Intervalle $(\nu, \nu+I)$ :

und es sei:

$$
f(x)=c_{v} \text { in }(y, y+\mathrm{I}) \quad(v=0, \mathrm{I}, 2, \ldots)
$$

$$
f(\mathrm{o})=c_{0}, \quad f(\nu)=\frac{\mathrm{I}}{2}\left(c_{\nu-1}+c_{\nu}\right) \quad(\nu=\mathrm{I}, 2, \ldots)
$$

Dann hat $f(x)$ die Eigenschaft A) in jedem Punkte $x_{0}$. Wegen:

$$
\int_{1}^{+\infty}\left|\frac{f(x)}{x}\right| d x=\sum_{\nu=1}^{\infty}\left|e_{\nu}\right| \int_{\nu}^{\nu+1} \frac{d x}{x}=\sum_{\nu=1}^{\infty}\left|e_{\nu}\right| \lg \frac{\nu+\mathrm{I}}{\nu}
$$

1 Dieser Satz findet sich, wie mich Herr HILB freundlichst anfmerksam machte, bereits bei H. WEYL, Jahresber. Math. Ver. 20 (I9I I), S. I34.

2 Das folgende Beispiel verdanke ich Herrn W. WIRTINGER. Ein andres Beispiel findet man bei A. Pringsheim, Math. Ann. 7 I (i9i I), S. 296. 
wird $f(x)$ die Eigenschaft B) dann und nur dann haben, wenn die Reihe $\sum_{\nu=1}^{\infty}\left|c_{\nu}\right| \lg \frac{\nu+1}{\nu}$ konvergiert, oder, was dasselbe heisst, wenn die Reihe

$$
\sum_{v=1}^{\infty}\left|c_{v}\right| \cdot \frac{\mathrm{I}}{v}
$$

konvergiert.

Falls die durch (12) gegebene Funktion $\varphi(\mu)$ existiert, so muss sein:

(I4) $\varphi(\mu)=2 \int_{0}^{+\infty} f^{\prime}(x) \cos \mu x d x=2 \sum_{\nu=0}^{\infty} c_{v} \int_{v}^{\nu+1} \cos \mu x d x=4 \frac{\sin \frac{\mu}{2}}{\mu} \sum_{\nu=0}^{\infty} c_{v} \cos \left(\nu+\frac{\mathrm{I}}{2}\right) \mu$

und diese letzte Reihe muss konvergent sein. Setzen wir also $c_{v}=\mathrm{I}$, wenn $v$ das Quadrat einer natürlichen Zahl ist: $\nu=k^{2}(k=1,2, \ldots)$, sonst aber $c_{\nu}==0$, so wird:

$$
\sum_{\nu=1}^{\infty}\left|c_{\nu}\right| \cdot \frac{\mathrm{I}}{\nu}=\sum_{k=1}^{\infty} \frac{\mathrm{I}}{k^{2}}
$$

so dass die Reihe (I3) konvergiert; die Funktion $f(x)$ hat also dann die Eigenschaften A) und B). Die in (14) auftretende Reihe aber wird: $\sum_{k=1}^{\infty} \cos \left(k^{2}+\frac{1}{2}\right) \mu$ und ist als trigonometrische Reihe, deren Koeffizienten nicht gegen o konvergieren, für alle $\mu$ abgesehen von einer Nullmenge divergent. ${ }^{1}$ Für die Funktion $f(x)$ gilt also das Fouriersche Integraltheorem (I I) nicht, weil die darin auftretende Funktion $\varphi(\mu)$ (abgesehen von einer Nullmenge) nicht existiert. Unsre Formel (Io) hingegen ist zur Darstellung von $f\left(x_{0}\right)$ anwendbar, und zwar ist in ihr $\Psi(\mu)=\mathrm{o}$ und:

$$
\Phi(\mu)=2 \sum_{k=1}^{\infty} \int_{k^{2}}^{k^{2}+\mathrm{i}} \frac{\sin \mu x}{x} d x
$$

welche Reihe offenbar konvergiert. Wie man sieht, entsteht sie aus der in (I4) auftretenden (divergenten) Reihe $2 \sum_{k=1}^{\infty} \int_{k^{3}}^{k^{2}+1} \cos \mu x d x$ durch gliedweise Integration nach $\mu$ von o bis $\mu$.

\footnotetext{
1 Vgl, z. B. H. Lebesaue, Leçons sur les séries trigọnométriques, S. I Io.
} 
Obwohl, wie wir eben sahen, die Tragweite in Formel (Io) eine grössere ist, als die der Fourierschen Integralformel (I I), so kann doch in manchen Fällen aus der Giltigkeit von (ro) auf die Giltigkeit von (I I) geschlossen werden, wodurch man zu sehr allgemeinen Kriterien für die Giltigkeit der Fourierschen Integralformel gelangt. Wir zeigen zunächst:

Satz II. Gilt für eine Funktion $f(x)$ Formel (10), existieren ferner fïr alle $\mu \geqq 0$ (abgesehen von einer Nullmenge) die durch (I2) definierten Funktionen $\varphi(\mu)$ und $\psi(\mu)$, und bestehen zwischen diesen und den durch (7) definierten Funktionen $\Phi(\mu)$ und $\Psi(\mu)$ die Beziehungen:

$$
\Phi(\mu)=\int_{0}^{\mu} \varphi(\mu) d \mu ; \quad \Psi(\mu)=\int_{0}^{\mu} \psi(\mu) d \mu \quad(\mu \geqq 0)
$$

so gilt für $f(x)$ auch die Fouriersche Integralformel (I I).

In der Tat, auf grund von (I5) kann geschrieben werden:

$$
\begin{aligned}
\int_{0}^{\lambda} \cos \mu x_{0} d \Phi(\mu)= & \int_{0}^{\lambda} \cos \mu x_{0} \varphi(\mu) d \mu \\
& \int_{0}^{\lambda} \sin \mu x_{0} d \Psi(\mu)=\int_{0}^{\lambda} \sin \mu x_{0} \psi(\mu) d \mu
\end{aligned}
$$

so dass (Io) unmittelbar in (I I) übergeht.

Erinnern wie uns an Satz I, so sehen wir, dass die Eigenschaften A) und B), zusammen mit den Beziehungen ( $\mathrm{I}_{5}$ ) für die Giltigkeit der Fourierschen Integralformel (I I) hinreichen.

Ein Beispiel zu diesem sehr allgemeinen Kriterium liefert die Funktion $\frac{\sin x^{1}}{x}$, die offenbar die Eigenschaften A) und B) hat. Hier ist $\Psi(\mu)=0$ und:

1 Wie A. Pringsheim (Math. Ann. 68 (I9Io), S. 368) mitteilt, wurde er von H. Weber aufmerksam gemacht, dass für die Funktion $\frac{\sin x}{x}$ die Fouriersche Integralformel gilt, während sie keiner der üblichen Bedingungen für die Giltigkeit dieser Formel genügt. 
(I7) $\quad \varphi(\mu)=2 \int_{0}^{+\infty} \frac{\sin x \cos \mu x}{x} d x=$

$$
\int_{0}^{+\infty} \frac{\sin (\mathrm{I}+\mu) x}{x} d x+\int_{0}^{+\infty} \frac{\sin (\mathrm{I}-\mu) x}{x} d x= \begin{cases}\pi & (\mathrm{O} \leqq \mu<\mathrm{I}) \\ x & (\mu-\mathrm{I}) \\ 2 & (\mu>\mathrm{I}) \\ 0 & \end{cases}
$$

Die zweite Formel (15) ist in trivialer Weise erfüllt $(\Psi(\mu)=0, \psi(\mu)=0)$. Um die erste Formel (I5) zu beweisen, beachten wir, dass:

$$
2 \int_{i}^{+\infty} \frac{\sin x \cos \mu x}{x} d x=\int_{(1+\mu) b}^{+\infty} \frac{\sin x}{x}-d x \pm \int_{|1-\mu| b}^{+\infty} \frac{\sin x}{x} d x
$$

Das Integral $\int_{b}^{+\infty} \frac{\sin x \cos \mu x}{x} d x$ bleibt also für alle $b$ und alle $\mu$ zwischen endlichen Grenzen. Daher ist:

$$
\begin{aligned}
\int_{0}^{\mu} \varphi(\mu) d \mu & =2 \int_{0}^{\mu}\left(\lim _{b \rightarrow+\infty} \int_{0}^{b} \frac{\sin x \cos \mu x}{x} d x\right) d \mu \\
& =2 \lim _{b \rightarrow+\infty} \int_{0}^{\mu}\left(\int_{0}^{b} \frac{\sin x \cos \mu x}{x} d x\right) d \mu=2 \lim _{b \rightarrow+\infty} \int_{0}^{b}\left(\int_{0 .}^{\mu} \frac{\sin x \cos \mu x}{x} d \mu\right) d x \\
& =2 \int_{0}^{+\infty} \frac{\sin x}{x} \cdot \frac{\sin \mu x}{x} d x=\Phi(\mu)
\end{aligned}
$$

womit auch die erste Formel (I5) bewiesen ist. - Aus (17) entnehmen wir, dass hier:

$$
\Phi(\mu)= \begin{cases}\pi \mu & (\mathrm{o} \leqq \mu \leqq \mathrm{I}) \\ \pi & (\mu \geqq \mathrm{I})\end{cases}
$$

die Fouriersche Integralformel lautet hier einfach:

$$
\frac{\sin x_{0}}{x_{0}}=\int_{0}^{1} \cos \mu x_{0} d \mu
$$




\section{$\$ 4$.}

Satz II kann noch verallgemeinert werden:

Satz III. Gilt fiir eine Funktion $f(x)$ Formel (10), sind die durch (7) definierten Funktionen $\Phi(\mu)$ und $\Psi(\mu)$ stetig für $\mu \geqq 0$, existieren für alle $\mu \geqq 0$ (ab. gesehen von einer Nullmenge) die durch (12) definierten Funktionen $p(\boldsymbol{\mu})$ und $\psi(\mu)$, bestehen für jedes Intervall ( $0 \leqq) \alpha \leqq \mu \leqq \beta$, das keinen Punkt einer gewissen reduziblen ${ }^{1}$ Punktmengs $Q$ enthält, die Beziehungen:

$$
\boldsymbol{\Phi}(\beta)-\boldsymbol{\Phi}(\alpha)=\int_{\alpha}^{\beta} \varphi(\mu) d \mu ; \quad \Psi(\beta)-\Psi(\alpha)=\int_{\alpha}^{\beta} \psi(\mu) d \mu,
$$

so gilt fiir $f(x)$ auch die Fouriersche Integralformel (11).

Wir haben zu zeigen, dass auch unter den Voraussetzungen von Satz III die Formeln (16) gelten. Dabei wird es genügen, anzunehmen, dass ins Intervall $[0, \lambda]$ nur ein einziges Punkt $q$ der Menge $Q$ fällt, da der Übergang zum allgemeinen Falle nach ganz bekannten Schlüssen vor sich geht; und zwar wollen wir etwa annehmen, der Punkt $q$ falle ins Innere von $[0, \lambda]$. Dann ist, wegen der Stetigkeit von $\boldsymbol{\Phi}(\mu)$ :

$$
\int_{0}^{j} \cos \mu x_{0} d \Phi(\mu)=\lim _{h \rightarrow+1} \int_{0}^{q} \cos \mu x_{0} d \Phi(\mu)+\lim _{h \rightarrow+0} \int_{q+h}^{j} \cos \mu x_{0} d \Phi(\mu) .
$$

In jedem der beiden Intervalle $[0, q-h]$ und $[q+h, \lambda]$ ist nun aber nach Voraussetzung $\boldsymbol{\Phi}(\mu)$ unbestimmtes Integral der integrierbaren Funktion $\varphi(\mu)$, so dass:

$$
\int_{0}^{q-h} \cos \mu x_{0} d \Phi(\mu)=\int_{0}^{q-h} \cos \mu x_{0} \varphi(\mu) d \mu ; \quad \int_{q+h}^{\lambda} \cos \mu x_{0} d \Phi(\mu)=\int_{q+h}^{\lambda} \cos \mu x_{0} \varphi(\mu) d \mu .
$$

Ess ist also:

${ }^{1}$ d. h. einer Punktmenge $Q$, die eine leere Ableitung $Q^{(v)}$ (von endlicher oder transfiniter Ordnung v) hat. Das Wort seduzibel ist also hier in dem Sinne gebraucht, in dem es von G. CANTOR ursprünglich gebraucht wurde. 


$$
\int_{0}^{\lambda} \cos \mu x_{0} d \Phi(\mu)=\lim _{h \rightarrow+0} \int_{0}^{q-h} \cos \mu x_{0} \varphi(\mu) d \mu+\lim _{h \rightarrow+0} \int_{q+h}^{\lambda} \cos \mu x_{0} \varphi(\mu) d \mu .
$$

Es existiert also der rechts stehende Grenzwert, d. h. das (eventuell) uneigentliche Integral $\int_{0}^{\lambda} \cos \mu x_{0} \varphi(\mu) d \mu$, und es ist:

$$
\int_{0}^{\lambda} \cos \mu x_{0} d \Phi(\mu)=\int_{0}^{\lambda} \cos \mu x_{0} \varphi(\mu) d \mu
$$

womit die erste Formel (I6) bewiesen ist; und ganz ebenso beweist man die zweite; nur ist festzuhalten, dass nun die auf den rechten Seiten der Formeln (I6) auftretenden Integrale uneigentliche Integrale (mit der singulären Stelle $q$ ) sein können.

Ebenso wird im allgemeinen Falle das in (I I) auftretende Integral ein uneigentliches Lebesguesches Integral sein können, dessen singuläre Punkte die Punkte von $Q$ und deren Häufungspunkte sind.

Aus Satz III entnehmen wir, dass die Fouriersche Integralformel fiir jede Funktion $f(x)$ gilt, die die Eigenschaften A) und B) hat, für die (fïr $\mu \geqq 0$ ) die Funktionen $\varphi(\mu)$ und $\psi(\mu)$ abgesehen von einer Nullmenge existieven, und in jedem Intervalle $[\alpha, \beta]$, das keinen Punkt einer gewissen reduziblen Menge Q enthält, die Beziehungen (I 8 ) gelten.

Als Beispiel betrachten wir die Funktion:

$$
f(x)=g(x) \cos q x \quad(q>0)
$$

wo $g(x)$ die Figenschaft B) habe und im Unendlichen monoton sei (d. h. es gebe ein $a$, sodass $g(x)$ für $x>a$ und $x<-a$ monoton ist). Offenbar ist dann:

$$
\lim _{x \rightarrow+\infty} g(x)=0 ; \quad \lim _{x \rightarrow-\infty} g(x)=0 .
$$

Die Funktion $f(x)$ hat dann ebenfalls die Eigenschaft B). Wir nehmen an, sie habe auch die Eigenschaft $\mathbf{A}$ ). Nach Satz I gilt dann für $f(x)$ Formel (Io) und $\boldsymbol{\Phi}(\mu)$ und $\Psi(\mu)$ sind (für $\mu \geqq 0$ ) stetig.

Wir untersuchen die zugehörige Funktion $\varphi(\mu)$ (für $\mu \geqq 0)$. Wählen wir $b>a$ und $p>b$, so ist nach dem zweiten Mittelwertsatz: 


$$
\begin{aligned}
\int_{b}^{p} f(x) \cos \mu x d x & =\int_{b}^{p} g(x) \cos q x \cos \mu x d x= \\
& =\frac{\mathrm{I}}{2} \int_{b}^{p} g(x)(\cos (\mu+q) x+\cos (\mu-q) x) d x= \\
& =\frac{\mathrm{I}}{2} g(b) \int_{b}^{p^{*}}(\cos (\mu+q) x+\cos (\mu-q) x) d x,
\end{aligned}
$$

und somit für $\mu \neq q$ :

$$
\left|\int_{b}^{p} f(x) \cos \mu x d x\right| \leqq|g(b)|\left(\frac{\mathrm{I}}{\mu+q}+\frac{1}{|\mu-q|}\right) .
$$

Ganz ebenso findet man:

$$
\left|\int_{-p}^{-b} f(x) \cos \mu x d x\right| \leqq|g(-b)|\left(\frac{\mathrm{I}}{\mu+q}+\frac{\mathrm{I}}{|\mu-q|}\right)
$$

Wegen (I9) folgt daraus, dass für $\mu \geqq 0$ und $\neq q$ das Integral:

$$
\varphi(\mu)=\int_{-\infty}^{+\infty} f(x) \cos \mu x d x
$$

existiert, und dass die Konvergenz von:

$$
\varphi_{n}(\mu)=\int_{-n}^{n} f(x) \cos \mu x d x
$$

gegen $\varphi(\mu)$ in jedem Intervalle (o@) $\alpha \leqq \mu \leqq \beta$, das den Punkt $q$ nicht enthält, gleichmässig erfolgt. Darum folgt weiter für jedes solche Intervall:

$$
\begin{aligned}
\int_{\alpha}^{\beta} \varphi(\mu) d \mu=\lim _{n \rightarrow \infty} \int_{\alpha}^{\beta} \varphi_{n}(\mu) d \mu=\lim _{n \rightarrow \infty} \int_{-n}^{n}\left(f(x) \int_{\alpha}^{\beta} \cos \mu x d \mu\right) d x \\
=\int_{-\infty}^{+\infty} f(x) \frac{\sin \beta x-\sin \alpha x}{x} d x=\Phi(\beta)-\Phi(\alpha) .
\end{aligned}
$$


Ganz ebenso finden wir, dass $\psi(\mu)$ für $\mu \geqq 0$ und $\neq q$ existiert und dass für jedes Intervall $(o \leqq) \alpha \leqq \mu \leqq \beta$, das den Punkt $q$ nicht enthält, die zweite Formel (18) gilt.

Für die Funktion $f(x)=g(x) \cos q x$ sind also die sämtlichen Voraussetzungen von Satz III erfüllt; wir sehen also, dass für sie die Fouriersche Integralformel (II) gilt, wobei aber das Integral auf der rechten Seite von (II) ein uneigentliches mit der singulären Stelle $q$ sein kann. - Ganz dasselbe gilt, wie analoge Rechnungen zeigen, für $f(x)=g(x) \sin q x$.

\section{$\S 5$.}

Das eben behandelte Beispiel ist ein Spezialfall des folgenden Satzes, der gleichfalls in Satz III enthalten ist:

Satz IV. Sei $f(x)$ eine Funktion der Eigenschaft A), die die Gestalt hat:

$$
f(x)=g(x) h(x)
$$

wo $g(x)$ eine im Unendlichen monotone Funktion der Eigenschaft B) und $h(x)$ eine periodische Funktion bedeutet. Dann gilt fïr $f(x)$ die Fouriersche Integralformel (I I).

Sei $g(x)$ etwa monoton für $x>a$ und $x<-a$. Ohne Beschränkung der Allgemeinheit können wir annehmen, $h(x)$ habe die Periode $2 \pi$.

Zunächst überzeugen wir uns leicht, dass auch $f(x)$ die Eigenschaft B) hat. In der Tat ist für $b>a$ :

$$
\int_{b}^{+\infty}\left|\frac{f(x)}{x}\right| d x \leqq \sum_{k=0}^{\infty}\left|\frac{g(b+2 k \pi)}{b+2 k \pi}\right| \cdot \int_{b}^{b+2 \pi}|h(x)| d x
$$

Da aber:

$$
\sum_{k=1}^{\infty}\left|\frac{g(b+2 k \pi)}{b+2 k \pi}\right| \leqq \frac{\mathrm{I}}{2 \pi} \int_{b}^{+\infty}\left|\frac{g(x)}{x}\right| d x
$$

ist, so folgt aus Eigenschaft B) von $g(x)$ die Konvergenz der in (2 I) auftretenden Reihe, und damit die Eigenschaft B) für $f(x)$.

Nach Satz I gilt also für $f(x)$ Formel (Io) und $\boldsymbol{\Phi}(\mu)$ und $\Psi(\mu)$ sind (für $\mu \geqq 0)$ stetig.

Sodann zeigen wir: Zu jedem $\delta>0$ gibt es ein $M$, so dass für alle lnter40-2661. Acta mathematica. 49. Imprimé le 29 juillet 1926. 
valle $[\xi, \eta]$ und alle $\mu \geqq 0$, die keinem der Intervalle $|\mu-k|<\delta(k=0,1,2, \ldots)$ angehören :

$$
\left|\int_{\xi}^{\eta} h(x) \cos \mu x d x\right|<M, \quad\left|\int_{\xi}^{\eta} h(x) \sin \mu x d x\right|<M .
$$

Sei etwa $\eta>\xi$ und sei die ganze Zahl $n$ so gewählt, dass $\xi+2 n \pi \leqq \eta<\xi+$ $+2(n+\mathrm{I}) \pi$. Dann ist:

$$
\int_{\xi}^{\eta} h(x) e^{i \mu x} d x=\sum_{k=0}^{n-1} \int_{\xi}^{\xi+2 \pi} h(x) e^{i \mu(x+2 k \pi)} d x+\int_{\xi+2 n \pi}^{\eta} h(x) e^{i \mu x} d x
$$

Da hierin:

$$
=\int_{\xi}^{\xi+2 \pi} h(x) e^{i \mu x} d x \cdot \frac{e^{2 \pi i \mu n}-\mathrm{I}}{e^{2 \pi i \mu}-\mathrm{I}}+\int_{\xi+2 n \pi}^{\eta} h(x) e^{i \mu x} d x
$$

$$
\left|\int_{\xi}^{\xi+2 \pi} h(x) e^{i \mu x} d x\right| \leqq \int_{\xi}^{\xi+2 \pi}|h(x)| d x, \quad\left|\int_{\xi+2 n \pi}^{\eta} h(x) e^{i \mu x} d x\right| \leqq \int_{\xi}^{\xi+2 \pi}|h(x)| d x
$$

ist (22) bewiesen. ${ }^{1}$

$$
\left|e^{2 \pi i \mu n}-\mathrm{I}\right| \leqq 2 \text {, }
$$

Wir gehen nun über zur Untersuchung von $\varphi(\mu)$. Sei $\mu>0, b>a, p>b$. Nach dem zweiten Mittelwertsatze ist:

$$
\int_{b}^{p} f(x) \cos \mu x d x=\int_{b}^{p} g(x) h(x) \cos \mu x d x=g(b) \int_{b}^{p^{*}} h(x) \cos \mu x d x
$$

Da auch hier die Beziehungen (I9) gelten, entnehmen wir aus (22): Ist $\varepsilon>0$ beliebig gegeben, und enthält das Intervall $(o<) \alpha \leqq \mu \leqq \beta$ keine natürliche Zahl, so gibt es ein $b_{0}$, so dass für alle $b \geqq b_{0}$, alle $p>b$ und alle $\mu$ von $[\alpha, \beta]$ :

$$
\left|\int_{b}^{p} f(x) \cos \mu x d x\right|<\varepsilon ; \quad\left|\int_{-p}^{-b} f(x) \cos \mu x d x\right|<\varepsilon
$$

1 Dieser einfache Beweis wurde mir von Herrn W. Wirtinger mitgeteilt. 
Daraus folgt sofort die Existenz von $\varphi(\mu)$ für $\mu \neq 0,1,2, \ldots$ und die gleichmässige Konvergenz der durch (20) definierten Funktion $\varphi_{n}(\mu)$ gegen $\varphi(\mu)$ in jedem Intervalle $(0<) \alpha \leqq \mu \leqq \beta$, das keine natürliche Zahl enthält. Daraus folgt wie in $\S 4$, dass in jedem solchen Intervalle die erste Gleichung (18) gilt, und ebenso zeigt man dies für die zweite.

Für die Funktion $f(x)=g(x) h(x)$ sind also sämtliche Voraussetzungen von Satz III erfüllt (wobei $Q$ die Menge der Zahlen $o, \mathrm{I}, 2, \ldots$ ist), und damit ist Satz IV bewiesen. Das in der Fourierschen Integralformel (I I) für $f(x)$ auftretende Integral wird hier im allgemeinen ein uneigentliches sein mit den singulären Stellen $\mathrm{O}, \mathrm{I}, 2, \ldots$

Ein verwandter Satz ist der folgende ${ }^{1}$ :

Satz IV a. Satz IV bleibt richtig, wenn darin unter $h(x)$ ein Funktion der Gestalt:

$$
h(x)=\sum_{v=1}^{\infty}\left(a_{v} \cos q_{v} x+b_{v} \sin q_{v} x\right)
$$

verstanden wird, vorausgesetzt dass die Reihe $\sum_{v=1}^{\infty}\left(\left|a_{v}\right|+\left|b_{v}\right|\right)$ konvergiert und die nicht-negativen Zahlen $q_{v}$ eine reduzible Menge $Q$ bilden.

Da hier $h(x)$ beschränkt ist, folgt aus der Eigenschaft B) von $g(x)$ sofort auch die Eigenschaft B) für $f(x)$. Weiter ist (wenn $\mu \geqq 0$ und von allen $q_{v}$ verschieden ist):

$$
\begin{aligned}
& \int_{\xi}^{\eta} h(x) \cos \mu x d x=\sum_{v=1}^{\infty}\left(a_{\nu} \int_{\xi}^{\eta} \cos q_{v} x \cos \mu x d x+b_{\nu} \int_{\xi}^{\eta} \sin q_{v} x \cos \mu x d x\right) \\
& =\frac{\mathrm{I}}{2} \sum_{\nu=1}^{\infty} \frac{a_{v}}{q_{v}+\mu}\left(\sin \left(q_{v}+\mu\right) \eta-\sin \left(q_{v}+\mu\right) \xi\right)+\frac{\mathrm{I}}{2} \sum_{\nu=1}^{\infty} \frac{a_{v}}{q_{v}-\mu}\left(\sin \left(q_{v}-\mu\right) \eta-\sin \left(q_{v}-\mu\right) \xi\right) \\
& -\frac{\mathrm{I}}{2} \sum_{v=1}^{\infty} \frac{b_{v}}{q_{v}+\mu}\left(\cos \left(q_{v}+\mu\right) \eta-\cos \left(q_{v}+\mu\right) \xi\right)-\frac{\mathrm{I}}{2} \sum_{\nu=1}^{\infty} \frac{b_{v}}{q_{v}-\mu}\left(\cos \left(q_{v}-\mu\right) \eta-\cos \left(q_{v}-\mu\right) \xi\right)
\end{aligned}
$$

und somit:

${ }^{1}$ Dieser Satz findet sich im Wesentlichen schon bei A. PringsheIM, Math. Ann. 68 (1910), S. $367-408 ; 71$ (I9II), S. $289^{--298}$. 


$$
\left|\int_{\xi}^{\eta} h(x) \cos \mu x d x\right| \leqq \sum_{\nu=1}^{\infty}\left(\left|a_{\nu}\right|+\left|b_{v}\right|\right)\left(\frac{\mathrm{I}}{q_{v}+\mu}+\frac{\mathrm{I}}{\left|q_{v}-\mu\right|}\right)
$$

und dieselbe Ungleichung erhält man für $\left|\int_{\xi}^{\eta} h(x) \sin \mu x d x\right|$. Wir entnehmen daraus, dass hier die Ungleichungen (22) gelten für alle Intervalle $[\xi, \eta]$ und alle $\mu \geqq 0$, die keinem der Intervalle $\left|\mu-q_{\nu}\right|<\delta(\nu=1,2, \ldots)$ angehören. Wie beim Beweise von Satz IV folgt daraus weiter, dass $\varphi(\mu)$ und $\psi(\mu)$ für jedes $\mu$ existieren, das weder Punkt noch Häufungspunkt von $Q$ ist, und dass die Beziehungen ( 18$)$ in jedem Intervalle $[\alpha, \beta]$ gelten, das weder einen Punkt noch einen Häufungspunkt von $Q$ enthält. Damit sind aber wieder alle Voraussetzungen von Satz III als erfüllt nachgewiesen, und somit ist auch Satz IV a bewiesen.

$\S 6$.

Ersetzen wir nun Eigenschaft B) durch die folgende:

C) Es sei:

$$
\lim _{x \rightarrow+\infty} \frac{f(x)}{x}=0 ; \quad \lim _{x \rightarrow-\infty} \frac{f(x)}{x}=0
$$

und es gebe ein $a$, so dass $\frac{f(x)}{x}$ monoton ist für $x>a$ und $x<-a$.

Wir erkennen leicht, dass (I) auch für jede Funktion $f(x)$ der Eigenschaften A) und C) gilt. In der Tat ist dann (wenn $a>\left|x_{0}\right|$ gewählt wurde) auch $\frac{f(x)}{x-x_{0}}$ monoton für $x>a$ und $x<-a$, und es ist auch:

$$
\lim _{x \rightarrow+\infty} \frac{f(x)}{x-x_{0}}=0 ; \quad \lim _{x \rightarrow-\infty} \frac{f(x)}{x-x_{0}}=0 .
$$

Nach dem zweiten Mittelwertsatze ist für $q>a, p>q$ :

$$
\left|\int_{q}^{p} f(x) \frac{\sin \lambda\left(x-x_{0}\right)}{x-x_{0}} d x\right|=\left|\frac{f(q)}{q-x_{0}} \int_{q}^{p^{*}} \sin \lambda\left(x-x_{0}\right) d x\right| \leqq \frac{2}{\lambda}\left|\frac{f(q)}{q-x_{0}}\right|
$$


ist also $\varepsilon>0$ beliebig gegeben und bedentet $\lambda_{0}$ irgend eine positive Zahl, so gibt es ein $b$, so dass für $p>q \geqq b$ und alle $\lambda \geqq \lambda_{0}$ :

$$
\left|\int_{q}^{p} f(x) \frac{\sin \lambda\left(x-x_{0}\right)}{x-x_{0}} d x\right|<\varepsilon, \quad\left|\int_{-p}^{-q} f(x) \frac{\sin \lambda\left(x-x_{0}\right)}{x-x_{0}} d x\right|<\varepsilon .
$$

Es existiert also das Integral $\int_{-\infty}^{+\infty} f(x) \frac{\sin \lambda\left(x-x_{0}\right)}{x-x_{0}} d x$, und es ist für $\lambda \geqq \lambda_{0}$ :

$$
\left|\int_{b}^{+\infty} f(x) \frac{\sin \lambda\left(x-x_{0}\right)}{x-x_{0}} d x\right| \leqq \varepsilon ; \quad\left|\int_{-\infty}^{-b} f(x) \frac{\sin \lambda\left(x-x_{0}\right)}{x-x_{0}} d x\right| \leqq \varepsilon
$$

und indem man von hieraus weiter schliesst wie in $\$$ I, beweist man die Giltigkeit von (I).

Man könnte daher versucht sein zu glauben, dass. Formel (Io) auch für alle Funktionen $f(x)$ der Eigenschaften A) und C) gilt. Das ist aber nicht der Fall. Sei etwa $f(x)=|x|^{\frac{1}{2}}$. Dann hat $f(x)$ die. Eigenschaften A) und C). Nach (7) ergibt sich $\boldsymbol{\Phi}(\mathrm{o})=\mathrm{o}$ und:

$$
\Phi(\mu)=2 \int_{0}^{+\infty} \frac{\sin \mu x}{x^{\frac{1}{2}}} d x=2 \mu^{-\frac{1}{2}} \int_{0}^{+\infty} \frac{\sin x}{x^{\frac{1}{2}}} d x \quad \text { für } \mu>0
$$

Das Integral $\int_{0}^{+\infty} \cos \mu x_{0} d \Phi(\mu)$ hat also hier keinen Sinn. Wohl aber werden wir im Folgenden zeigen, dass ( Io) für jede Funktion $f(x)$ der Eigenschaft A) gilt, die im Unendlichen beschränkt und monoton ist. Wir schicken folgende Bemerkungen voran.

Wir setzen im Folgenden stets:

$$
\Phi(\mu)=\lim _{n \rightarrow \infty} \int_{-n}^{n} f(x) \frac{\sin }{x} \frac{\mu x}{x} d x
$$


(25) $\Psi(\mu)=\int_{-1}^{1} f(x) \frac{\mathrm{I}-\cos \mu x}{x} d x-\lim _{n \rightarrow \infty}\left(\int_{1}^{n} f(x) \frac{\cos \mu x}{x} d x+\int_{-n}^{-1} f(x) \frac{\cos \mu x}{x} d x\right)$,

vorausgesetzt, dass diese Grenzwerte existieren. Für jede beliebige ungerade Funktion $f(x)$ ist dann $\boldsymbol{\Phi}(\mu)=0$, für jede gerade Funktion $f(x)$ ist $\Psi(\mu)=0$. Abgesehen von diesen Spezialfällen werden wir es aber nur mit dem Falle zu haben, dass die Integrale:

$$
\int_{0}^{+\infty} f(x) \frac{\sin \mu x}{x} d x, \int_{-\infty}^{0} f(x) \frac{\sin \mu x}{x} d x, \int_{1}^{+\infty} f(x) \frac{\cos \mu x}{x} d x, \int_{-\infty}^{-1} f(x) \frac{\cos \mu x}{x} d x
$$

existieren. Die Grenzwerte (24) und (25) sind dann sicher vorhanden.

Die durch (25) definierte Funktion $\Psi(\mu)$ unterscheidet sich von der bisher verwendeten, durch $(7)$ definierten, falls sie alle beide existieren, nur durch eine additive Konstante, auf die es nicht ankommt, da die Funktion $\Psi(\mu)$ nur in der Verbindung $\int \sin \mu x_{0} d \Psi(\mu)$ auftritt.

$\$ 7$.

Wir werden nun zeigen (§§ 7-I6), dass Formel (Io) für jede Funktion der Eigenschaft A) gilt, die im Unendlichen beschränkt und monoton ist.

Sei zunächst $f(x)$ eine Funktion der Eigenschaft $\mathrm{A}$ ), die im Unendlichen monoton gegen o konvergiert:

$$
\lim _{x \rightarrow+\infty} f(x)=0 ; \quad \lim _{x \rightarrow-\infty} f(x)=0
$$

Wir untersuchen zuerst die zugehörige Funktion $\Phi(\mu)$. Ist $f(x)$ monoton für $x>a$ und $x<-a$, so haben wir nach dem zweiten Mittelwertsatz für $p>q>a$, wenn $M$ eine geeignete Konstante bedeutet:

$$
\left|\int_{q}^{p} f(x) \frac{\sin \mu x}{x} d x\right|=\left|f(q) \int_{q}^{p^{*}} \frac{\sin \mu x}{x} d x\right|=\left|f(q) \int_{\mu q}^{\mu p^{*}} \frac{\sin x}{x} d x\right| \leqq|f(q)| . M .
$$

Wegen (26) gibt es also zu jedem $\varepsilon>0$ ein $b$, so dass für $b \leqq q<p$ und alle $\mu$ : 


$$
\left|\int_{q}^{p} f(x) \frac{\sin }{x} \frac{\mu x}{x} d x\right|<\varepsilon
$$

Daraus folgt: Für alle $\mu$ existiert das Integral $\int_{0}^{+\infty} f(x) \underset{x}{\sin \mu x} d x$, und das Integral $\int_{0}^{n} f(x) \frac{\sin \frac{\mu x}{x}}{x} d x$ konvergiert mit $n \rightarrow \infty$ gleichmässig für alle $\mu$ gegen $\int_{0}^{+\infty} f(x) \frac{\sin }{x} \frac{\mu x}{x} d x$. Dies letztere Integral ist also eine stetige Funktion von $\mu$. Da genau dasselbe von $\int_{-\infty}^{0} f(x) \frac{\sin }{x} \frac{\mu x}{x} d x$ gilt, so sehen wir: $\Phi(\mu)$ existiert und ist stetig fïr alle $\mu \geqq 0$.

Nun untersuchen wir die Funktion $\Psi(\mu)$. Wie vorhin erhalten wir:

$$
\left|\int_{q}^{p} f(x) \frac{\cos \mu x}{x}-d x\right|=\left|f(q) \int_{\mu q}^{\mu p^{*}} \frac{\cos x}{x} d x\right|
$$

woraus wir entnehmen: zu jedem $\varepsilon>0$ und $\delta>0$ gibt es ein $b$, so dass für $b \leqq q<p$ und $\mu \geqq \delta$ :

$$
\left|\int_{q}^{p} f(x) \frac{\cos \mu x}{x} d x\right|<\varepsilon
$$

Daraus folgt: für alle $\mu>0$ existiert $\int_{i}^{+\infty} f(x) \frac{\cos \mu x}{x} d x$, und für alle

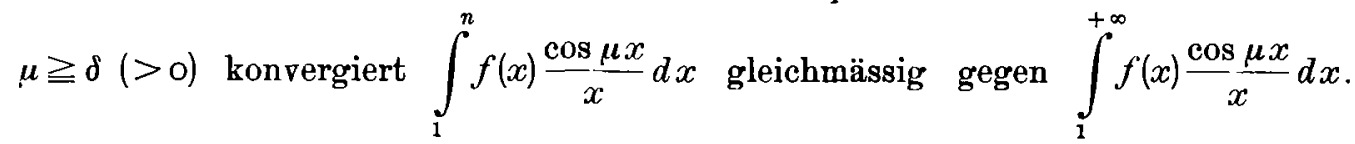
Analoges gilt für $\int_{-\infty}^{-1} f(x) \frac{\cos \mu x}{x} d x$, so dass wir sehen: $\Psi(\mu)$ existiert und ist stetig fiir alle $\mu>0$.

Zugleich haben wir bewiesen: setzen wir: 
(27)

$$
\begin{aligned}
\boldsymbol{\Phi}_{n}(\mu) & =\int_{-n}^{n} f(x) \frac{\sin \frac{\mu x}{x}}{x} d x \\
\Psi_{n}(\mu) & =\int_{-1}^{1} f(x) \frac{1-\cos \mu x}{x} d x-\int_{1}^{n} f(x) \frac{\cos \mu x}{x} d x-\int_{-n}^{-1} f(x) \frac{\cos \mu x}{x} d x
\end{aligned}
$$

so konvergiert $\Phi_{u}(\mu)$ gleichmässig für alle $\mu \geqq 0$ gegen $\Phi(\mu)$ und $\Psi_{n}(\mu)$ gleichmässig für alle $\mu \geqq \delta(>0)$ gegen $\Psi(\mu)$.

Wie wir in $\$ 6$ gesehen haben, gilt auch hier Formel (1). Da ferner:

$$
\int_{-\infty}^{+\infty} f(x) \frac{\sin \lambda\left(x-x_{0}\right)}{x-x_{0}} d x=\int_{-\infty}^{+\infty} f\left(x_{0}+x\right) \frac{\sin \lambda x}{x} d x
$$

ist dieses Integral nichts anderes als die zur Funktion $f\left(x_{0}+x\right)$ gehörige Funktion $\boldsymbol{\Phi}(\lambda)$, und daher, nach dem eben Bewiesenen stetig für alle $\lambda \geqq 0$.

Ganz wie in $\S$ I finden wir nun für $0<h<\lambda$ :

$$
\begin{gathered}
\int_{-\infty}^{+\infty} f(x) \frac{\sin \lambda\left(x-x_{0}\right)}{x-x_{0}} d x-\int_{-\infty}^{+\infty} f(x)-\frac{\sin \left(x-x_{0}\right)}{x-x_{0}} d x= \\
\lim _{n \rightarrow \infty} \int_{-n}^{n}\left(f(x) \int_{h}^{\lambda} \cos \mu\left(x-x_{0}\right) d \mu\right) d x=\lim _{n \rightarrow \infty} \int_{h}^{\lambda}\left(\cos \mu x_{0} d \Phi_{n}(\mu)+\sin \mu x_{0} d \Psi_{n}(\mu)\right) \\
=\int_{h}^{\lambda}\left(\cos \mu x_{0} d \Phi(\mu)+\sin \mu x_{0} d \Psi(\mu)\right) .
\end{gathered}
$$

Wegen der Stetigkeit des Integrales (28) konvergiert für $h \rightarrow 0$ auf der linken Seite der Subtrahend gegen o. Wir haben daher:

$$
\int_{\infty}^{+\infty} f(x) \frac{\sin \lambda\left(x-x_{0}\right)}{x-x_{0}} d x=\lim _{h \rightarrow+0} \int_{h}^{\lambda}\left(\cos \mu x_{0} d \Phi(\mu)+\sin \mu x_{0} d \Psi(\mu)\right)
$$

und wenn wir schreiben: 
(29) $\lim _{h \rightarrow+0} \int_{h}^{\lambda}\left(\cos \mu x_{0} d \Phi(\mu)+\sin \mu x_{0} d \Psi(\mu)\right)=\int_{0}^{\lambda}\left(\cos \mu x_{0} d \Phi(\mu)+\sin \mu x_{0} d \Psi(\mu)\right)$

so können wir den Satz aussprechen:

Satz V. Fï jede Funktion $f(x)$ der Eigenschaft A), die im Unendlichen monoton gegen o konvergiert, gilt Formel (10); darin ist die durch (24) definierte Funktion $\boldsymbol{\Phi}(\mu)$ stetig fiir $\mu \geqq 0$, die durch (25) definierte Funktion $\Psi(\mu)$ stetig fiir $\mu>0$ und das in (10) auftretende Integral ist im Sinne von (29) zu verstehen.

Um auch hier den Utbergang zur Fourierschen Integralformel (II) zu vollziehen, betrachten wir die durch (12) definierte Funktion $\varphi(\mu)$. Wir haben hier für $a<q<p$ :

$$
\left|\int_{q}^{p} f(x) \cos \mu x d x\right|=\left|f(q) \int_{q}^{p^{*}} \cos \mu x d x\right| \leqq{ }_{\mu}^{2}|f(q)|
$$

woraus wir in gewohnter Weise schliessen, dass $\varphi(\mu)$ für $\mu>0$ existiert, und dass die durch (20) definierte Funktion $\varphi_{n}(\mu)$ für $\mu \geqq \delta(>0)$ gleichmässig gegen $\varphi(\mu)$ konvergiert; es ist also $\varphi(\mu)$ für $\mu>0$ stetig, und dasselbe gilt für $\psi(\mu)$. Ganz wie in $\$ 4$ können wir auch schliessen, dass in jedem Intervalle $o<\alpha \leqq \mu \leqq \beta$ die Relationen (I8) bestehen. Daraus folgt für $o<h<\lambda$ :

$$
\int_{h}^{\lambda}\left(\cos \mu x_{0} d \Phi(\mu)+\sin \mu x_{0} d \Psi(\mu)\right)=\int_{h}^{\lambda}\left(\cos \mu x_{0} \varphi(\mu)+\sin \mu x_{0} \psi(\mu)\right) d \mu .
$$

Vollzieht man noch den Grenzübergang $h \rightarrow+0$, so wird aus (Io) die Fouriersche Integralformel (I I), in der nun aber das auftretende Integral ein uneigentliches (mit der singulären Stelle $\mu=0$ ) sein kann. Wir haben also ${ }^{1}$ :

Satz V a. Für jede Funktion $f(x)$ der Eigenschaft $\mathbf{A}$ ), die im Unendlichen monoton gegen o konvergiert, gilt die Fouriersche Integralformel (I I).

1 Dieser Satz stammt von A. Pringsheim, l. c., not. I S. 315.

41-2661. Acta mathematica. 49. Imprimé le 29 juillet 1926. 
$\S 8$.

Sei nun

$$
f(x)=\mathrm{I} .
$$

Dann wird $\Psi(\mu)=$ o und:

(30)

$$
\boldsymbol{\Phi}(\mu)=\int_{-\infty}^{+\infty} \frac{\sin \mu}{x} d x= \begin{cases}0 & \text { für } \mu=0 \\ \pi & \text { für } \mu>0 .\end{cases}
$$

Es ist also für jedes $\lambda>0$ :

$$
\int_{0}^{\lambda}\left(\cos \mu x_{0} d \Phi(\mu)+\sin \mu x_{0} d \Psi(\mu)\right)=\pi,
$$

woraus man ersieht: fïr die Funktion $f(x)=\mathrm{I}$ gilt Formel (IO); es ist in ihr $\boldsymbol{\Phi ( \mu )}$ die durch (3o) gegebene unstetige Funktion, und $\Psi(\mu)=0$. Die Fouriersehe Integralformel (I I) verliert natürlich hier jeden Sinn.

Sei sodann $f(x)$ die Funktion:

$$
f_{0}(x)=\left\{\begin{aligned}
1 & \text { für } x>0 \\
0 & \text { für } x=0 \\
-1 & \text { für } x<0
\end{aligned}\right.
$$

Hier wird $\boldsymbol{\Phi}(\mu)=0$, und (für $\mu>0$ ):

$$
\begin{aligned}
\Psi(\mu) & =2 \int_{0}^{1} \frac{\mathrm{I}-\cos \mu x}{x} d x-2 \int_{1}^{+\infty} \frac{\cos \mu x}{x} d x \\
& =2 \int_{0}^{1} \frac{\mathrm{I}-\cos \mu x}{x} d x-2 \int_{\mu}^{+\infty} \frac{\cos x}{x} d x .
\end{aligned}
$$

Es ist also:

$$
\Psi^{\prime}(\mu)=2 \int_{0}^{1} \sin \mu x d x+2 \frac{\cos \mu}{\mu}=\frac{2}{\mu},
$$

und somit (für $\mu>0$ ):

Es ist also für o $<h<\lambda$ :

$$
\Psi(\mu)=\lg \mu^{2}+C
$$




$$
\int_{h}^{\lambda} \sin \mu x_{0} d \Psi(\mu)=2 \int_{n}^{\lambda} \frac{\sin \mu x_{0}}{\mu} d \mu \text {. }
$$

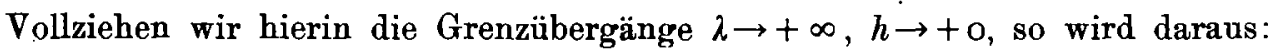

$$
\int_{0}^{+\infty} \sin \mu x_{0} d \Psi(\mu)=2 \int_{0}^{+\infty} \frac{\sin \mu}{\mu} x_{0} d \mu=\left\{\begin{aligned}
\pi & \text { für } x_{0}>0 \\
0 & \text { für } x_{0}=0 \\
-\pi & \text { für } x_{0}<0
\end{aligned}\right.
$$

Daraus ersehen wir: auch für die Funktion (3 I) gilt Formel (Io); das in ihr auftretende Integral $\int_{0}^{+\infty}$ ist dabei im Sinne des Grenzwertes $\lim _{h \rightarrow+0} \int_{h}^{+\infty}$ zu verstehen. Zugleich sehen wir, dass das bekannte Integral $\frac{2}{\pi} \int_{0}^{+\infty} \frac{\sin \mu x_{0}}{\mu} d \mu$ in gewissem Sinne als (verallgemeinerte) Fouriersche Integraldarstellung der Funktion (3 I) aufgefasst werden kann.

Nun können wir ganz allgemein den Satz aussprechen:

Satz VI. Für jede im Unendlichen beschränkte und monotone Funktion $f(x)$ der Eigenschaft A) gilt Formel (1о); darin bedeutet $\Phi(\mu)$ eine fïr $\mu \geqq 0$ definierte und fïr $\mu>0$ stetige Funktion, die den Grenzwert $\Phi(+0)$ besitzt, während $\Psi(\mu)$ fïr $\mu>0$ definiert und stetig ist; das in (1о) auftretende Integral ist im folgenden Sinne zu verstehen:

$$
\int_{0}^{1} \cos \mu x_{0} d \boldsymbol{\Phi}(\mu)+\lim _{h \rightarrow+0} \int_{h}^{1} \sin \mu x_{0} d \Psi(\mu)+\int_{1}^{+\infty}\left(\cos \mu x_{0} d \Phi(\mu)+\sin \mu x_{0} d \Psi(\mu)\right) .
$$

In der Tat, da $f(x)$ im Unendlichen monoton und beschränkt ist, existieren endliche Grenzwerte:

$$
\lim _{x \rightarrow+\infty} f(x)=c_{1}, \quad \lim _{x \rightarrow-\infty} f(x)=c_{2}
$$

Wir bezeichnen wieder mit $f_{0}(x)$ die Funktion (3I) und setzen:

$$
f(x)=\frac{c_{1}+c_{9}}{2}+\frac{c_{1}-c_{2}}{2} f_{0}(x)+f_{1}(x)
$$


Dann ist $f_{1}(x)$ im Unendlichen monoton mit:

$$
\lim _{x \rightarrow+\infty} f_{1}(x)=0, \quad \lim _{x \rightarrow-\infty} f_{1}(x)=0
$$

Nach Satz V und den zu Beginn dieses Paragrafen gefundenen Resultaten ist jeder der drei Summanden auf der rechten Seite von (32) der Darstellung (IO fähig; daraus erhält man sofort Satz VI: Offenbar ist, wenn man (30) beachtet:

$$
\Phi(+o)=\frac{c_{1}+c_{2}}{2} \pi
$$

\section{$\$ 9$.}

Sei nun:

$$
f(x)=\cos q x \quad(q>0)
$$

Dann ist $\Psi(\mu)==0$ und:

$$
\begin{aligned}
\boldsymbol{\Phi}(\mu) & =\int_{-\infty}^{+\infty} \cos q x \frac{\sin \mu x}{x} d x \\
& =\frac{1}{2} \int_{-\infty}^{+\infty} \frac{\sin (\mu+q) x}{x} d x+\frac{1}{2} \int_{-\infty}^{+\infty} \frac{\sin (\mu-q) x}{x} d x= \begin{cases}0 & \text { für } o \leqq \mu<q \\
\frac{\pi}{2} & \text { für } \mu=q \\
x & \text { für } \mu>q .\end{cases}
\end{aligned}
$$

Es ist also für jedes $\lambda>q$ :

$$
\int_{0}^{\lambda}\left(\cos \mu x_{0} d \Phi(\mu)+\sin \mu x_{0} d \Psi(\mu)\right)=\pi \cos q x_{0}
$$

d. h. fuir die Funktion $\cos q x(q>0)$ gilt Formel (Io); es ist in ihr $\Phi(\mu)$ die durch (33) gegebene unstetige Funktion, und $\Psi(\mu)=0$.

Sei sodann:

$$
f(x)=\sin q x \quad(q>0)
$$

Dann ist $\Phi(\mu)=0$ und:

$$
\Psi(\mu)=\int_{-1}^{1} \sin q x \frac{\mathrm{I}-\cos \mu x}{x} d x-\int_{1}^{+\infty} \sin q x \frac{\cos \mu x}{x} d x-\int_{-\infty}^{-1} \sin q x \frac{\cos \mu x}{x} d x
$$




$$
\begin{aligned}
& =\int_{-1}^{1} \frac{\sin q x}{x} d x-\int_{-\infty}^{+\infty} \frac{\sin q x \cos \mu x}{x} d x \\
& =\int_{-1}^{1} \frac{\sin q x}{x} d x-\frac{1}{2} \int_{-\infty}^{+\infty} \frac{\sin (q+\mu) x}{x} d x-\frac{1}{2} \int_{-\infty}^{+\infty} \frac{\sin (q-\mu) x}{x} d x,
\end{aligned}
$$

mithin:

$$
\Psi(\mu)=\int_{-1}^{1} \frac{\sin q x}{x} d x- \begin{cases}\pi & \text { für } 0 \leqq \mu<q \\ \frac{\pi}{2} & \text { für } \mu=q \\ 0 & \text { für } \mu>q .\end{cases}
$$

Es ist also für jedes $\lambda>q$ :

$$
\int_{0}^{\lambda}\left(\cos \mu x_{0} d \Phi(\mu)+\sin \mu x_{0} d \Psi(\mu)\right)=\pi \sin q x_{0},
$$

d. h. für die Funktion $\sin q x(q>0)$ gilt Formel (10); es ist in ihr $\Phi(\mu)=0$ und $\Psi(\mu)$ die unstetige Funktion (34).

$\S$ Io.

Wir bezeichnen wieder mit $f_{0}(x)$ die Funktion $(31)$, setzen

$$
f(x)=f_{0}(x) \cos q x \quad(q>0)
$$

und wollen zeigen, dass auch für diese Funktion Formel (Io) gilt. Zunächst beweisen wir die Giltigkeit der Formel (1). Für $\left|x_{0}\right|<\xi<\eta$ ist:

$$
\begin{aligned}
& \int_{x_{0}+5}^{x_{0}+\eta} f(x) \frac{\sin \lambda\left(x-x_{0}\right)}{x-x_{0}} d x=\int_{5}^{\eta} f\left(x_{0}+x\right) \frac{\sin \lambda x}{x} d x=\int_{5}^{\eta} \cos q\left(x_{0}+x\right) \frac{\sin \lambda x}{x} d x \\
& =\frac{1}{2} \cos q x_{0}\left(\int_{\xi}^{\eta} \frac{\sin (\lambda+q) x}{x} d x+\int_{\xi}^{\eta} \frac{\sin (\lambda-q) x}{x} d x\right)+ \\
& +\frac{1}{2} \sin q x_{0}\left(\int_{5}^{\eta} \frac{\frac{\cos (\lambda+q) x}{x}}{x} d x-\int_{5}^{\eta} \frac{\cos (\lambda-q) x}{x} d x\right) .
\end{aligned}
$$


Wir entnehmen daraus: zu jedem $\varepsilon>0$ und $\delta>0$ gibt es ein $b$, so dass für $b \leqq \xi<\eta$ und alle der Bedingung $|\lambda-q| \geqq \delta$ genügenden nicht-negativen $\lambda$ die Ungleichung gilt:

$$
\left|\int_{x_{0}+\xi}^{x_{0}+\eta} f(x) \frac{\sin \lambda\left(x-x_{0}\right)}{x-x_{0}} d x\right|<\varepsilon
$$

Da Analoges für $\int_{x_{0}-\eta}^{x_{0}-\bar{j}} f(x) \frac{\sin \lambda\left(x-x_{0}\right)}{x-x_{0}} d x$ gilt, sehen wir, dass für alle nichtnegativen $\lambda \neq q$ das Integral (I) existiert, und dass für $\lambda \geqq q+\delta$ die Ungleichung gilt:

$$
\left|\int_{x_{0}+b}^{+\infty} f(x) \frac{\sin \lambda\left(x-x_{0}\right)}{x-x_{0}} d x\right| \leqq \varepsilon
$$

Indem man von hier aus weiter schliesst, wie in $\S$ I, beweist man die Giltigkeit von (I).

Das Verhalten des Integrales (I) an der Stelle $\lambda=q$ wird charakterisiert durch die Beziehung:

$$
\lim _{h \rightarrow 0}\left(\int_{-\infty}^{+\infty} f(x) \frac{\sin (q+h)\left(x-x_{0}\right)}{x-x_{0}} d x-\int_{-\infty}^{+\infty} f(x) \frac{\sin (q-h)\left(x-x_{0}\right)}{x-x_{0}} d x\right)=0
$$

In der Tat, jedenfalls ist (für jedes $b>0$ ):

(36) $\lim _{h \rightarrow 0}\left(\int_{x_{0}-b}^{x_{0}+b} f(x) \frac{\sin (q+h)\left(x-x_{0}\right)}{x-x_{0}} d x-\int_{x_{0}-b}^{x_{0}+b} f(x) \frac{\sin (q-h)\left(x-x_{0}\right)}{x-x_{0}} d x\right)=0$.

Ferner ist für $b>\left|x_{0}\right|$ :

$$
\begin{aligned}
\int_{x_{0}+b}^{+\infty}+\int_{-\infty}^{x_{0}-b} f(x) \frac{\sin (q+h)\left(x-x_{0}\right)-\sin (q-h)\left(x-x_{0}\right)}{x-x_{0}} d x \\
\quad=\int_{b}^{+\infty}\left(\cos q\left(x_{0}+x\right)-\cos q\left(x_{0}-x\right)\right) \frac{\sin (q+h) x-\sin (q-h) x}{x} d x
\end{aligned}
$$


Über eine Verallgemeinerung der Fourierschen Integralformel.

$$
\begin{aligned}
& =-4 \sin q x_{0} \int_{b}^{+\infty} \frac{\sin q x \cos q x \sin h x}{x} d x \\
& =\sin q x_{0}\left(\int_{b}^{+\infty} \frac{\cos (2 q+h) x}{x} d x-\int_{b}^{+\infty} \frac{\cos (2 q-h) x}{x} d x\right) .
\end{aligned}
$$

Daraus entnehmen wir: zu jedem $\varepsilon>0$ gibt es ein $b$, so dass für $0<h \leqq q$ :

$$
\left|\int_{x_{0}+b}^{+\infty}+\int_{-\infty}^{x_{0}-b} f^{\prime}(x) \frac{\sin (q+h)\left(x-x_{0}\right)-\sin (q-h)\left(x-x_{0}\right)}{x-x_{0}} d x\right|<\varepsilon .
$$

Diese Ungleichung zusammen mit (36) aber ergibt die Behauptung (35).

Nun wenden wir uns zur Untersuchung der zu unsrer F'unktion $f(x)$ gehörigen Funktionen $\boldsymbol{\Phi}(\mu)$ und $\Psi(\mu)$. Da $f(x)$ ungerade, ist $\boldsymbol{\Phi}(\mu)=$ o. Für $\mu \geqq 0$ und $\neq q$ ist:

$$
\begin{aligned}
\Psi(\mu)=2 \int_{0}^{1} \cos q x \frac{\mathrm{I}-\cos \mu x}{x} d x-2 \int_{1}^{+\infty} \cos q x \frac{\cos \mu x}{x} d x \\
=2 \int_{0}^{1} \cos q x \frac{\mathrm{I}-\cos \underline{\mu x}}{x} d x-\int_{1}^{+\infty}\left(\frac{\cos (\mu+q) x}{x}+\frac{\left.\cos \frac{(\mu-q) x}{x}\right)}{x}\right) d x \\
=2 \int_{0}^{1} \cos q x \frac{\mathrm{I}-\frac{\cos \mu x}{x}}{x} d x-\int_{\mu+q}^{+\infty} \frac{\cos x}{x} d x-\int_{|\mu-q|}^{+\infty} \frac{\cos x}{x} d x
\end{aligned}
$$

woraus sich ergibt:

$$
\Psi^{\prime}(\mu)=2 \int_{0}^{1} \cos q x \sin \mu x d x+\frac{\cos (\mu+q)}{\mu+q}+\frac{\cos (\mu-q)}{\mu-q}=\frac{2 \mu}{\mu^{2}-q^{2}},
$$

und somit:

$$
\Psi(\mu)=\lg \left|\mu^{2}-q^{2}\right|+C .
$$

Für das Verhalten von $\Psi(\mu)$ an der Stelle $q$ folgt hieraus:

$$
\lim _{h \rightarrow 0}(\Psi(q+h)-\Psi(q-h))=0 .
$$


Betrachten wir die durch (27) definierte Funktion $\Psi_{n}(\mu)$, so ist:

$$
\Psi_{n}(\mu)-\Psi(\mu)=2 \int_{n}^{+\infty} \cos q x \frac{\cos \mu x}{x} d x=\int_{n(\mu+q)}^{+\infty} \frac{\cos x}{x} d x+\int_{n|\mu-q|}^{+\infty} \frac{\cos x}{x} d x
$$

woraus sich ergibt, dass $\Psi_{n}(\mu)$ gleichmässig gegen $\Psi(\mu)$ konvergiert in jedem Intervalle (o@) $\alpha \leqq \mu \leqq \beta$, das den Punkt $q$ nicht enthält.

Nun ist nach Schlüssen, die schon wiederholt durchgefübrt wurden, für

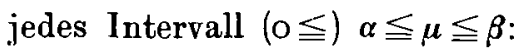

$$
\int_{-\infty}^{+\infty} f(x) \frac{\sin \beta\left(x-x_{0}\right)}{x-x_{0}} d x-\int_{-\infty}^{+\infty} f(x) \frac{\sin \alpha\left(x-x_{0}\right)}{x-x_{0}} d x=\lim _{n \rightarrow \infty} \int_{\alpha}^{\beta} \sin \mu x_{0} d \Psi_{n}(\mu)
$$

mithin wenn $[\alpha, \beta]$ den Punkt $q$ nicht enthält:

$$
\int_{-\infty}^{+\infty} f(x) \frac{\sin \beta\left(x-x_{0}\right)}{x-x_{0}} d x-\int_{-\infty}^{+\infty} f(x) \frac{\sin \alpha\left(x-x_{0}\right)}{x-x_{0}} d x=\int_{\alpha}^{\beta} \sin \mu x_{0} d \Psi(\mu) .
$$

Für $\lambda>q$ und $o<h<q$ erhalten wir, indem wir diese Formel auf die Intervalle $[0, q-h]$ und $[q+h, \lambda]$ anwenden:

$$
\begin{array}{r}
\int_{-\infty}^{+\infty} f^{\prime}(x) \frac{\sin }{x-x_{0}} d x-\left(\int_{-\infty}^{+\infty} f(x) \frac{\sin (q+h)\left(x-x_{0}\right)}{x-x_{0}} d x-\int_{-\infty}^{+\infty} f(x) \frac{\sin (q-h)\left(x-x_{0}\right)}{x-x_{0}} d x\right) \\
=\int_{0}^{q-h} \sin \mu x_{0} d \Psi(\mu)+\int_{q+h}^{\lambda} \sin \mu x_{0} d \Psi(\mu)
\end{array}
$$

Vollziehen wir hierin den Grenzübergang $h \rightarrow+0$ und setzen:

$$
\lim _{h \rightarrow+0}\left(\int_{0}^{q-h} \sin \mu x_{0} d \Psi(\mu)+\int_{q+h}^{\lambda} \sin \mu x_{0} d \Psi(\mu)\right)=\int_{0}^{\lambda} \sin \mu x_{0} d \Psi(\mu)
$$

so erhalten wir bei Beachtung von (35):

$$
\int_{-\infty}^{+\infty} f(x) \frac{\sin \lambda\left(x-x_{0}\right)}{x-x_{0}} d x=\int_{0}^{\lambda} \sin \mu x_{0} d \Psi(\mu)
$$


Und da, wie bewiesen, Formel (I) gilt, folgt daraus durch den Grenzübergang $\lambda \rightarrow+\infty$ der Satz:

Bedeutet $f_{0}(x)$ die Funktion (3I), so gilt für $f_{0}(x) \cos q x(q>0)$ die Formel (1o), in deren Integral gemäss (39) an der Stelle $\lambda=q$ der Cauchysche Hauptwert zu nehmen ist; es ist in ihr $\Phi(\mu)=0$ und $\Psi(\mu)$ die Funktion (38).

Wegen (37) kann diese Formel auch so geschrieben werden:

$$
\int_{0}^{+\infty} \sin \mu x \frac{\mu}{\mu^{2}-q^{2}} d \mu=\left\{\begin{array}{cc}
\frac{\pi}{2} \cos q x & \text { für } x>0 \\
0 & \text { für } x=0 \\
-\frac{\pi}{2} \cos q x & \text { für } x<0
\end{array}\right.
$$

wobei links wieder an der Stelle $q$ der Cauchysche Hauptwert zu nehmen ist. Formel (40), die natürlich auch durch direkte Rechnung gewonnen werden kann, ist in gewissem Sinne die Fouriersche Integraldarstellung der auf ihrer rechten Seite stehenden Funktion.

\section{§ I I.}

In ganz derselben Weise kann der Fall:

$$
f(x)=f_{0}(x) \sin q x \quad(q>0)
$$

behandelt werden, wo wieder $f_{0}(x)$ die Funktion (3I) bedeutet. Ganz wie in $\$$ Io beweist man die Giltigkeit der Formeln (I) und (35).

Da diesmal $f(x)$ gerade, ist $\Psi(\mu)=0$. Für $\mu \geqq 0$ und $\neq q$ ist:

$$
\Phi(\mu)=2 \int_{0}^{+\infty} \sin q x \frac{\sin \mu x}{x} d x=2 \int_{0}^{1} \frac{\sin q x \sin \mu x}{x} d x+\int_{|\mu-q|}^{+\infty} \frac{\cos x}{x} d x-\int_{\mu+q}^{+\infty} \frac{\cos x}{x} d x
$$

woraus sich ergibt:

(4I) $\Phi^{\prime}(\mu)=2 \int_{0}^{1} \sin q x \cos \mu x d x+\frac{\cos (\mu+q)}{\mu+q}-\frac{\cos (\mu-q)}{\mu-q}=\frac{\mathrm{I}}{q+\mu}+\frac{1}{q-\mu}=\frac{2 q}{q^{2}-\mu^{2}}$, und somit:

$$
\Phi(\mu)=\lg \left|\frac{q+\mu}{q-\mu}\right|+C
$$

42-2661. Acta mathematica. 49. Imprimé le 30 juillet 1926. 
Für das Verhalten von $\Phi(\mu)$ an der Stelle $q$ gilt auch hier:

$$
\lim _{h \rightarrow 0}(\boldsymbol{\Phi}(q+h)-\boldsymbol{\Phi}(q-h))=0
$$

Indem man weiter schliesst, wie in $\$$ Io, findet man:

Bedeutet $f_{0}(x)$ die Funktion (31), so gilt für $f(x)=f_{0}(x) \sin q x(q>0)$ die Formel (1о), in deren Integral an der Stelle $\lambda=q$ der Cauchysche Hauptwert zu nehmen ist; es ist in ihr $\Phi(\mu)$ die Funktion $(42)$ und $\Psi(\mu)=0$.

Wegen (4I) kann diese Formel auch geschrieben werden:

$$
\int_{0}^{+\infty} \cos \mu x \frac{q}{q^{2}-\mu^{2}} d \mu=\left\{\begin{array}{c}
\frac{\pi}{2} \sin q x \text { für } x \geqq 0 \\
-\frac{\pi}{2} \sin q x \text { für } x \leqq 0
\end{array} \quad(q>0)\right.
$$

wobei links wieder an der Stelle $q$ der Cauchysche Hauptwert zu nehmen ist.

\section{$\S$ I 2.}

Nun nehmen wir $f(x)$ in der Form an:

$$
f_{1}(x)=g(x) \cos q x \quad \text { oder } \quad f_{z}(x)=g(x) \sin q x \quad(q>0)
$$

wo $g(x)$ im Unendlichen monoton gegen o konvergiere:

$$
\lim _{x \rightarrow+\infty} g(x)=0 ; \quad \lim _{x \rightarrow-\infty} g(x)=0
$$

Sei $g(x)$ monoton für $x>a$ und $x<-a$. Wir untersuchen die zu $f_{1}(x)$ gehörige Funktion $\boldsymbol{\Phi}(\mu)$ :

$$
\Phi^{(1)}(\mu)=\int_{-\infty}^{+\infty} g(x) \cos q x \frac{\sin \mu x}{x} d x
$$

Da für $a<\xi<\eta$ :

$$
\int_{\xi}^{\eta_{\xi}} g(x) \cos q x \frac{\sin \mu x}{x} d x=\frac{\mathrm{I}}{2} g(\xi)\left(\int_{\xi}^{\xi *} \frac{\sin (\mu+q) x}{x} d x+\int_{\xi}^{\xi *} \frac{\sin (\mu-q) x}{x} d x\right)
$$

gibt es zu jedem $\varepsilon>0$ ein $b$, so dass für $b \leqq \xi<\eta$ und alle $\mu \geqq 0$ : 


$$
\left|\int_{\xi}^{\eta} g(x) \cos q x \frac{\sin \mu x}{x} d x\right|<\varepsilon ;\left|\int_{-\eta}^{-\xi} g(x) \cos q x \frac{\sin \mu x}{x} d x\right|<\varepsilon .
$$

Daraus folgt: für alle $\mu$ existiert $\Phi^{(1)}(\mu)$, und die durch (27) definierte Funktion $\Phi_{n}(\mu)$ konvergiert für alle $\mu$ gleichmässig gegen $\Phi^{(1)}(\mu)$. Es ist also $\Phi^{(1)}(\mu)$ eine für alle $\mu \geqq 0$ definierte und stetige Funktion.

Nun untersuchen wir die zu $f_{1}(x)$ gehörige Funktion $\Psi(\mu)$ :

$$
\begin{aligned}
\Psi^{(1)}(\mu)=\int_{-1}^{1} g(x) \cos q x \frac{\mathrm{I}-\cos \frac{\mu x}{x}}{x} d x-\int_{1}^{+\infty} g(x) \cos q x \frac{\cos \mu x}{x} d x & \\
& -\int_{-\infty}^{-1} g(x) \cos q x \frac{\cos \mu x}{x} d x
\end{aligned}
$$

Hier haben wir für $a<\xi<\eta$ und $\mu \geqq 0$ und $\neq q$ :

$$
\begin{array}{r}
\int_{\xi}^{\eta} g(x) \cos q x \frac{\cos \mu x}{x} d x=\frac{\mathrm{I}}{2} g(\xi)\left(\int_{\xi}^{\xi^{*}} \frac{\cos (\mu+q) x}{x} d x+\int_{\xi}^{\xi_{\xi}^{*}} \frac{\cos (\mu-q) x}{x} d x\right) \\
=\frac{\mathrm{I}}{2} g(\xi)\left(\int_{(\mu+q) \xi}^{(\mu+q) \xi^{*}} \frac{\cos x}{x} d x+\int_{|\mu-q| \xi}^{|\mu-q| \xi^{*}} \frac{\cos x}{x} d x\right)
\end{array}
$$

und somit gibt es zu jedem $\varepsilon>0$ und $\delta>0$ ein $b$, so dass für $b \leqq \xi<\eta$ und $\mu \geqq 0,|\mu-q| \geqq \delta$ :

$$
\left|\int_{\xi}^{\eta} g(x) \cos q x \frac{\cos \mu x}{x} d x\right|<\varepsilon, \quad\left|\int_{\eta}^{-\xi} g(x) \cos q x \frac{\cos \mu x}{x} d x\right|<\varepsilon .
$$

Daraus folgt: für alle $\mu \geqq 0$ und $\neq q$ existiert $\Psi^{(1)}(\mu)$, und die durch (27) definierte Funktion $\Psi_{n}(\mu)$ konvergiert in jedem Intervalle $(0 \leqq) \alpha \leqq \mu \leqq \beta$, das den Punkt $q$ nicht enthält, gleichmässig gegen $\Psi^{(1)}(\mu)$. Es ist also $\Psi^{(1)}(\mu)$ eine für alle $\mu \geqq 0$ und $\neq q$ definierte und stetige Funktion.

Für das Verhalten von $\Psi^{(1)}(\mu)$ an der Stelle $q$ finden wir:

$$
\lim _{h \rightarrow 0}\left(\Psi^{(1)}(q+h)-\Psi^{(1)}(q-h)\right)=\mathrm{o}
$$


In der Tat, es ist:

$$
\begin{aligned}
\Psi^{(1)}(q+h)-\Psi^{(1)}(q-h)--\int_{-\infty}^{+\infty} g(x) \cos q x & \frac{\cos (q+h) x-\cos (q-h) x}{x} d x \\
& =-\frac{1}{2} \int_{\infty}^{+\infty} g(x) \frac{\cos (2 q+h) x-\cos (2 q-h) x}{x} d x .
\end{aligned}
$$

Bezeichnen wir die zu $g(x)$ gehörige Funktion $\Psi$ mit $\Psi^{(0)}(\mu)$, so ist also:

$$
\Psi^{(1)}(q+h)-\Psi^{(1)}(q-h)=\frac{\mathrm{I}}{2}\left(\Psi^{(0)}(2 q+h)-\Psi^{(0)}(2 q-h)\right)
$$

Da $g(x)$ im Unendlichen monoton gegen o konvergiert, ist aber zufolge $\$ 7 \Psi^{(0)}(\mu)$ stetig für $\mu>0$. Durch (44) ist also (43) bewiesen.

Völlig analoge Rechnungen ergeben für die zu $f_{\mathbf{2}}(x)=g(x) \sin q x$ gehörigen Funktionen $\boldsymbol{\Phi}(\boldsymbol{\mu})$ und $\Psi(\mu)$ : es ist $\boldsymbol{\Phi}^{\prime 2)}(\boldsymbol{\mu})$ definiert und stetig fï ${ }^{\cdot} \boldsymbol{\mu} \geqq 0$ und $\neq q$, wïhrend $\Psi^{(2)}(\mu)$ fïr alle $\mu \geqq 0$ definiert und stetig ist; und es ist:

$$
\lim _{h \rightarrow 0}\left(\boldsymbol{\Phi}^{(2)}(q+h)-\boldsymbol{\Phi}^{(2)}(q-h)\right)=0
$$

Nun wollen wir zeigen, dass auch für die jetzt betrachteten Funktionen $f_{1}(x)$ und $f_{2}(x)$, (falls sie die Eigenschaft A) haben) Formel (I) gilt. Wir setzen zu dem Zwecke:

$$
I^{(1)}(\lambda) \cdots \int_{-\infty}^{+\infty} f_{1}(x) \frac{\sin \lambda\left(x-x_{0}\right)}{x-x_{0}} d x ; \quad I^{(2)}(\lambda)==\int_{-\infty}^{+\infty} f_{2}(x) \frac{\sin \lambda\left(x-x_{0}\right)}{x-x_{0}} d x
$$

Wir führen die Untersuchung für $f_{1}(x)$ durch; für $f_{2}(x)$ geht sie analog vor sich. Es ist:

$$
\begin{aligned}
I^{(1)}(\lambda) & =\int_{-\infty}^{+\infty} g\left(x_{0}+x\right) \cos q\left(x_{0}+x\right) \frac{\sin \lambda x}{x} d x \\
& =\cos q x_{0} \int_{-\infty}^{+\infty} g\left(x_{0}+x\right) \cos q x \frac{\sin \lambda x}{x} d x-\sin q x_{0} \int_{-\infty}^{+\infty} g\left(x_{0}+x\right) \sin q x \frac{\sin \frac{\lambda x}{x} d x .}{x}
\end{aligned}
$$


Da ebenso wie $g(x)$ auch $g\left(x_{0}+x\right)$ im Unendlichen monoton gegen o konvergiert, folgt aus dem oben über $\Phi^{(1)}$ und $\Phi^{(2)}$ bewiesenen, dass das Integral

$$
\int_{-\infty}^{+\infty} g\left(x_{0}+x\right) \cos q x \frac{\sin \lambda x}{x} d x \quad \text { für alle } \lambda \geqq 0
$$

das Integral

$$
\int_{\infty}^{+\infty} g\left(x_{0}+x\right) \sin q x \frac{\sin \lambda x}{x} d x \quad \text { für alle } \lambda \geqq 0 \text { und } \neq q
$$

existiert und stetig ist. Es existiert also das Integral $I^{(1)}(\lambda)$ und ist stetig für alle $\lambda \geqq 0$ und $\neq q$. Hinsichtlich seines Verhaltens für $\lambda=q$ entnehmen wir aus (45):

$$
\lim _{h \rightarrow 0}\left(I^{(1)}(q+h)-I^{(1)}(q-h)\right)=\mathrm{o}
$$

Endlich zeigen wir mit Hilfe wiederholt durchgeführter Schlïsse: zu jedem $\varepsilon>0$ und $\delta>0$ gehört ein $b$, so dass für alle der Ungleichung $\lambda \geqq q+\delta$ genügenden $\lambda$ :

$$
\left|\int_{b}^{+\infty} f_{1}(x) \frac{\sin \lambda\left(x-x_{0}\right)}{x-x_{0}} d x\right|<\varepsilon ; \quad\left|\int_{\infty}^{-b} f_{1}(x) \frac{\sin \lambda\left(x-x_{0}\right)}{x-x_{0}} d x\right|<\varepsilon
$$

von hier aus schliesst man wie in $\S_{\mathrm{I}}$ : genügt $f_{1}(x)$ (oder $\left.f_{2}(x)\right)$ der Bedingung $\left.\mathbf{A}\right)$, so gilt fiii $f_{1}(x)$ (bzw. $\left.f_{2}(x)\right)$ die Formel (r).

Ganz wie in $\S$ Io erhalten wir nun den Satz: Bedeutet $g(x)$ eine im Unendlichen monoton gegen o konvergierende Funktion, so gilt fïr jede der beiden Funktionen $g(x) \cos q x$ und $g(x) \sin q x(q>0)$, falls sie die Eigenschaft A) hat, Formel (ro), in der an der. Stelle $\lambda=q$ der Cauchysche Hauptwert zu nehmen ist. ${ }^{1}$

Wie schon wiederholt, können wir hier weiter schliessen, dass unter den genannten Voraussetzungen auch die Fouriersche Integralformel (I I) gilt, in der wieder an der Stelle $\lambda-q$ der Cauchysche Hauptwert zu nehmen ist. ${ }^{2}$

1 Genauer gesprochen: da im Falle $g(x) \cos q x$ die Funktion $\Phi(\mu)$, im Falle $g(x) \sin q x$ die Funktion $\Psi(\mu)$ für $\mu=q$ stetig bleibt, muss im ersten Falle nur vom zweiten, im zweiten Falle nur vom ersten Summanden des Integranden $\cos \mu x_{0} d \Phi(\mu)+\sin \mu x_{0} d \Psi(\mu)$ der Cauchysche IIauptwert gebildet werden.

2. Pringsheim, Math. Ann. 68 (1910), S. 398, 399. 


\section{$\S 13$.}

Wir fassen nun die Resultate der $\$ \$ 9-$ i 2 zusammen. Sei:

$$
f(x)=g(x) \cos q x \quad(q>0)
$$

wo $g(x)$ im Unendlichen monoton und beschränkt sei. Wir setzen wie in (32):

$$
g(x)=\frac{c_{1}+c_{2}}{2}+\frac{c_{1}-c_{2}}{2} f_{0}(x)+g_{1}(x)
$$

wo $f_{0}(x)$ die Funktion (3I) bedeutet, und:

$$
e_{1}=\lim _{x \rightarrow+\infty} g(x), \quad e_{2}=\lim _{x \rightarrow \infty} g(x)
$$

dann konvergiert $g_{1}(x)$ im Unendlichen monoton gegen 0 .

Wir bezeichnen nun die zu $\cos q x, f_{0}(x) \cos q x, g_{1}(x) \cos q x$ gehörigen Funktionen $\Phi$ und $\Psi$ der Reihe nach mit $\Phi^{*}(\mu), \psi^{*}(\mu) ; \Phi^{* *}(\mu), \Psi^{* *}(\mu) ; \Phi^{* * *}(\mu), \Psi^{* * *}(\mu)$. Da $\Psi^{*}(\mu)=0$ und $\Phi^{* *}(\mu)=0$ ist, erhalten wir für die zu $f(x)$ gehörigen Funktionen $\boldsymbol{D}$ und $\Psi$ :

$$
\boldsymbol{D}(\mu)=\frac{c_{1}+c_{2}}{2} \boldsymbol{D}^{*}(\mu)+\boldsymbol{\Phi}^{* * *}(\mu) ; \quad \Psi(\mu)=\frac{c_{1}-c_{2}}{2} \Psi^{* *}(\mu)+\Psi^{* * *}(\mu)
$$

und die Resultate der $\$ \$ 9-12$ ergeben: $\Phi^{*}(\mu)$ ist die Funktion $(33) ; \Phi^{* * *}(\mu)$ ist stetig für $\mu \geqq 0 ; \Psi^{* *}(\mu)$ und $\Psi^{* * *}(\mu)$ sind stetig für $\mu \geqq 0$ und $\neq q$, und es ist:

$$
\lim _{h \rightarrow 0}\left(\Psi^{* *}(q+h)-\Psi^{* *}(q-h)\right)=0 ; \quad \lim _{h \rightarrow 0}\left(\Psi^{* * *}(q+h)-\Psi^{* * *}(q-h)\right)=0 ;
$$

und wir erhalten den Satz:

Satz VII. Fïr jede Funktion $f(x)$ der Figenschaft A), die die Gestalt hat $f(x)=g(x) \cos q x(q>0)$, wo $g(x)$ im Unendlichen beschränkt und monoton ist, gilt Formel (10):

$$
f\left(x_{0}\right)=\frac{1}{\pi}\left(\int_{0}^{+\infty} \cos \mu x_{0} d \Phi(\mu)+\sin \mu x_{0} d \Psi(\mu)\right)
$$

wo im zweiten Summanden an der Stelle $q$ der Cauchysche Hauptwert zu nehmen ist.

Genau so finden wir: 
Über eine Verallgemeinerung der Fourierschen Integralformel.

Satz VII a. Die Aussage vor Satz VII gilt auch für $f(x)=g(x) \sin q x(q>0)$, nur dass nun im ersten. Summanden an der Stelle $q$ der Cauchysche Hauptwert zu nehmen ist.

\section{$\S$ I 4 .}

Wir betrachten nun allgemein den Fall, dass $f(x)$ die Gestalt hat:

$$
f(x)=g(x) h(x)
$$

wo $g(x)$ im Unendlichen monoton gegen o konvergiert, und $h(x)$ eine periodische I inktion bedeutet.

Ohne Beschränkung der Allgemeinheit können wir annehmen, $h(x)$ habe die Periode $2 \pi$. Die Funktion $g(x)$ sei für $x>a$ monoton.

Wir untersuchen zunächst die zu $f(x)$ gehörige Funktion $\Phi(\mu)$. Sei:

$$
h(x){\underset{2}{2}}_{a_{0}}^{a_{k=1}}+\sum_{k}^{\infty}\left(a_{k} \cos k x+b_{k} \sin k x\right)
$$

die Fouriersche Reihe von $h(x)$. Sei $N$ irgend eine natürliche Zal. Wir setzen:

$$
h^{*}(x)=\frac{a_{0}}{2}+\sum_{k=1}^{N}\left(a_{k} \cos k x+b_{k} \sin k x\right)
$$

und nehmen die Zerlegung vor:

$$
h(x)=h^{*}(x)+h^{* *}(x)
$$

Entsprechend zerlegt sich dann $\Phi(\mu)$ in:

$$
\Phi(\mu)=\Phi^{*}(\mu)+\Phi^{* *}(\mu)
$$

Úber $\Phi^{*}(\mu)$ sind wir durch $\S 7$ und $\S$ I 2 völlig orientiert: es existiert $\Phi^{*}(\mu)$ und ist stetig für alle $\mu \geqq 0$ und $1,2, \ldots, N$, und es ist:

$$
\lim _{h \rightarrow 0}\left(\boldsymbol{\Phi}^{*}(k+h)-\boldsymbol{\Phi}^{*}(k-h)\right)=0 \quad(k=\mathrm{I}, 2, \ldots, N) .
$$

Wir haben noch $\Phi^{* *}(\mu)$ zu untersuchen.

Es ist nach dem zweiten Mittelwertsatze für $a<\xi<\eta$ :

$$
\int_{\xi}^{\eta} g(x) h^{* *}(x) \underset{x}{\sin \mu x} d x=\frac{g(\xi)}{\xi} \int_{\xi}^{\xi *} h^{* *}(x) \sin \mu x d x
$$


Nach (23) aber ist, wenn $n$ eine geeignete ganze Zahl bedeutet:

$$
\int_{\xi}^{\xi *} h^{* *}(x) e^{i \mu x} d x=\int_{\xi}^{\xi+2 \pi} h^{* *}(x) e^{i \mu x} d x \cdot e^{2 \pi i \mu n}-1
$$

und hierin ist:

$$
\left|e^{2 \pi i \mu n}-\mathrm{I}\right| \leqq 2 ; \quad\left|\int_{\xi i 2 n \pi}^{\xi *} h^{* *}(x) e^{i \mu x} d x\right| \leqq \int_{\xi}^{\xi+2 \pi}\left|h^{* *}(x)\right| d x .
$$

Ferner ist offenbar:

Es ist also

$$
\int_{\xi}^{\xi+2 \pi} h^{* *}(x) e^{i k x} d x=0 \quad(k=0, \mathrm{I}, 2, \ldots, N)
$$

$$
\int_{\xi}^{\xi+2 \pi} h^{* *}(x) e^{i \mu x} d x \cdot \frac{\mathrm{I}}{e^{2 \pi} i \mu^{2} \ldots-}
$$

eine analytische Funktion von $\mu$, die für $\mu=0,1,2, \ldots, N$ regulär ist. Aus der Periodizität von $e^{2 \pi i \mu}$ und der Ungleichung

$$
\left|\int_{\xi}^{\xi+2 \pi} h^{* *}(x) e^{i \mu x} d x\right| \leqq \int_{\xi}^{\xi+2 \pi}\left|h^{* *}(x)\right| d x
$$

folgt nun sofort: Zu jedem $\delta>0$ gibt es ein $M$, sodass für alle Intervalle $\left[\xi, \xi^{*}\right]$ und alle $\mu \geqq 0$, die keinem der Intervalle $|\mu-k|<\delta(k=N+\mathrm{I}, N+2, \ldots)$ angehören, die Ungleichungen gelten:

$$
\left|\int_{\xi}^{\xi} h^{* *}(x) \cos \mu x d x\right|<M ; \quad\left|\int_{\zeta}^{\xi *} h^{* *}(x) \sin \mu x d x\right|<M .
$$

Aus (47) ergibt sich nun: zu jedem $\varepsilon>0$ und $\delta>0$ gibt es ein $b$, sodass für $b \leqq \xi<\eta$ und für alle $\mu \geqq 0$, die keinem der Intervalle $|\iota-k|<\delta(k=N+\mathrm{I}, N+2 \ldots)$ angehören, die Ungleichungen gelten:

$$
\left|\int_{\xi}^{\eta} g(x) h^{* *}(x) \frac{\sin \mu x}{x} d x\right|<\varepsilon, \quad\left|\int_{-\eta}^{-\xi} g(x) h^{* *}(x) \frac{\sin \mu x}{x} d x\right|<\varepsilon .
$$


Daraus schliessen wir in bekannter Weise: die Funktion $\Phi^{* *}(\mu)$ existiert und ist stetig für alle $\mu \geqq 0$, die $\neq N+\mathrm{I}, N+2, \ldots$ sind.

Zusammen mit dem vorhin über $\Phi^{*}(\mu)$ festgestellten ergibt das, da in den obigen Ủberlegungen die natürliche Zahl $N$ ganz beliebig war: Die Funktion $\boldsymbol{\Phi}(\mu)$ existiert und ist stetig für alle $\mu \geqq 0$, die $\neq \mathrm{I}, 2, \ldots$ sind, und es ist:

$$
\lim _{h \rightarrow 0}(\boldsymbol{\Phi}(k+h)-\Phi(k-h))=\mathrm{o} \quad(k=\mathrm{I}, 2, \ldots)
$$

Ganz ebenso beweist man: Die Funktion $\Psi(\mu)$ existiert und ist stetig für alle $\mu>0$, die $\neq \mathrm{I}, 2, \ldots$ sind, und es ist:

$$
\lim _{h \rightarrow 0}(\Psi(k+h)-\Psi(k-h))=\mathrm{O} \quad(k=\mathrm{I}, 2, \ldots)
$$

Nun beweisen wir wieder die Giltigkeit der Formel (I). Das Integral:

$$
I(\lambda)=\int_{-\infty}^{+\infty} f(x) \frac{\sin \lambda\left(x-x_{0}\right)}{x-x_{0}} d x=\int_{-\infty}^{+\infty} f\left(x_{0}+x\right)^{\sin \lambda x} x d x
$$

ist die zu $f\left(x_{0}+x\right)$ gehörige Funktion $\boldsymbol{\Phi}$. Nach dem eben Bewiesenen existiert es also und ist stetig für $\lambda \geqq 0$ und $\neq \mathrm{I}, 2 \ldots$, und es ist:

$$
\lim _{h \rightarrow 0}(I(k+h)-I(k-h))=0 \quad(k=1,2, \ldots) .
$$

Ferner gibt es zu jedem $\varepsilon>0$ und $\delta>0$ ein $b$, sodass für alle $\lambda$, die keinem der Intervalle $|\lambda-k|<\delta(k=\mathrm{i}, 2, \ldots)$ angehören:

$$
\left|\int_{b}^{+\infty} f(x) \frac{\sin \lambda\left(x-x_{0}\right)}{x-x_{0}} d x\right|<\varepsilon, \quad\left|\int_{-\infty}^{b} f(x) \frac{\sin \lambda\left(x-x_{0}\right)}{x-x_{0}} d x\right|<\varepsilon .
$$

Also gilt hier, falls $f(x)$ die Eigenschaft A) hat, Formel (I) im folgenden Sinne: Bedeutet $\delta$ irgend eine positive Zahl und ist $\left\{\lambda_{v}\right\}$ eine Folge positiver Zahlen mit $\lim _{v \rightarrow \infty} \lambda_{v}=+\infty$, die keinem der Intervalle $|\lambda-k|<\delta(k=1,2, \ldots)$ angehören, so ist:

$$
f\left(x_{0}\right)=\frac{\mathrm{I}}{x} \lim _{\nu \rightarrow \infty} \int_{-\infty}^{+\infty} f(x) \frac{\sin \lambda_{v}\left(x-x_{0}\right)}{x-x_{0}} d x .
$$

43-2661. Acta mathematica. 49. Imprimé le 30 juillet 1926. 
Wieder gilt für jedes Intervall $0<\alpha \leqq \mu \leqq \beta$, das keinen der Punkte I, $2, \ldots$ enthält:

$$
I(\beta)-I(\alpha)=\int_{\alpha}^{\beta}\left(\cos \mu x_{0} d \Phi(\mu)+\sin \mu x_{0} d \Psi(\mu)\right)
$$

Sei nun $\lambda>0$ und $\neq 1,2, \ldots$ Ist die ganze Zahl $n$ so gewählt, dass $n<\lambda<n+\mathrm{I}$, und bedeuten $h_{0}, h_{1}, \ldots, h_{n}$ positiven Zahlen $<\frac{\mathrm{I}}{2}$, so gilt also:

$$
\begin{aligned}
I(\lambda)-\sum_{k=1}^{n}\left(I\left(k+h_{k}\right)-I\left(k-h_{k}\right)\right)-I\left(h_{0}\right)=\sum_{k=0}^{n-1} \int_{k+h_{k}}^{k+1}\left(\cos \mu x_{0} d \Phi(\mu)+\sin \mu x_{0} d \Psi(\mu)\right) \\
+\int_{n+h_{n}}^{h_{k+1}}\left(\cos \mu x_{0} d \Phi(\mu)+\sin \mu x_{0} d \Psi(\mu)\right) .
\end{aligned}
$$

Vollziehen wir hierin den Grenzübergang $h_{k} \rightarrow+0(k=0,1, \ldots, n)$, so erhalten wir wegen (49) und der Stetigkeit von $I(\lambda)$ für $\lambda=0$ :

$$
I(\lambda)=\int_{0}^{\lambda} \cos \mu x_{0} d \Phi(\mu)+\sin \mu x_{0} d \Psi(\mu)
$$

worin $\int_{0}^{\lambda}$ soviel bedeutet, wie $\lim _{h \rightarrow+0} \int_{h}^{\lambda}$, und an den Stellen $\lambda=x(k=z=1,2, \ldots, n) \operatorname{der}$ Cauchysche Hauptwert zu nehmen ist.

Beachten wir (50), so können wir also den Satz aussprechen:

Satz VIII. Fiir jede Funktion $f(x)$ der Eigenschaft A), die die Gestalt hat $f(x)=g(x) h(x)$, wo $g(x)$ im Unendlichen monoton gegen o konvergiert, und $h(x)$ eine Funktion der Periode $p$ bedeutet, gilt Formel (1о). Es ist in ihr $\int_{0}^{\lambda}$ im Sinne von $\lim _{h \rightarrow 0} \int_{h}^{\lambda} z u$ verstehen, für $\lambda=-\frac{2 k \pi}{p}(k=\mathrm{I}, 2, \ldots)$ der. Cauchysche Hauptwert zu nehmen, 
und der Grenzwert $\int_{0}^{+\infty}=\lim _{\lambda \rightarrow+\infty} \int_{0}^{\lambda} i n$ der Weise zu bilden, dass $\lambda$ ausserhalb der Intervalle $\left|\lambda-\begin{array}{c}2 k \pi \\ p\end{array}\right|<\delta(k=\mathrm{I}, 2, \ldots)$ bleibt, wo $\delta>0$ beliebig klein sein kann.

Auch hier kann der Utbergang zur Fourierschen Integralformel (I I) vollzogen werden. Es ist für $\alpha<\xi<\eta$ :

$$
\int_{\xi}^{\eta} f(x) \cos \mu x d x=g(\xi) \int_{\xi}^{\xi^{*}} h(x) \cos \mu x d x
$$

Aus (22) folgt also: zu jedem $\varepsilon>0$ und $\delta>0$ gibt es ein $b$, sodass für $b \leqq \xi<\eta$ und alle $\mu \geqq 0$, die keinem der Intervalle $|\mu-k|<\delta(k=0,1,2, \ldots)$ angehören:

$$
\left|\int_{\xi}^{\eta} f(x) \cos \mu x d x\right|<\varepsilon, \quad\left|\int_{-\eta}^{-\vdots} f(x) \cos \mu x d x\right|<\varepsilon .
$$

Daraus folgt, dass die durch (12) definierte Funktion $\varphi(\mu)$ für alle $\mu>0$ und $\neq \mathrm{I}, 2, \ldots$ existiert, und dass die durch $(20)$ definierte Funktion $\varphi_{n}(\mu)$ in jedem Intervalle $o<\alpha \leqq \mu \leqq \beta$, das keinen der Punkte I, $2, \ldots$ enthält, gleichmässig gegen $\varphi(\mu)$ konvergiert. Ganz analoges gilt für $\psi(\mu)$. In bekannter Weise folgt daraus, dass in jedem solchen Intervalle $[\alpha, \beta]$ die Formeln (I 8 ) gelten, woraus wir weiter schliessen:

Satz VIII a. Für jede Funktion $f^{\prime}(x)$ der Figenschaft A), die die Gestalt hat $f(x)=g(x) h(x)$, wo $g(x)$ im Unendlichen monoton gegen o konvergiert, und $h(x)$ eine Funktion der Periode $p$ bedeutet, gilt die Fouviersche Integralformel (I I); das in ihr auftretende Integral ist so zu verstehen wie in Satz VIII.

\section{§. I 5 .}

Nunmehr sei $f(x)$ selbst eine periodische Funktion. Ohne Beschränkung der

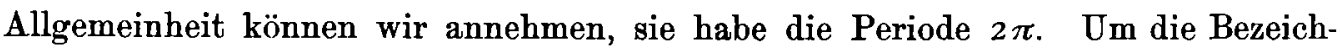
nungen von $\S$ I4 verwenden zu können, schreiben wir $f(x)=h(x)$, und zerlegen wie in $\S$ I $4 h(x)$ in $h^{*}(x)+h^{* *}(x)$, und bezeichnen die zugehörigen Funktionen $\Phi$ mit $\boldsymbol{\Phi}^{*}(\mu), \Phi^{* *}(\mu)$. 
Was $\Phi^{*}(\mu)$ anlangt, entnehmen wir unmittelbar aus $\$ \S 8,9$ :

$$
\text { (5 I) }\left\{\begin{array}{l}
\Phi^{*}(\mathrm{o})=\mathrm{o}, \Phi^{*}(\mu)=\pi \frac{a_{0}}{2} \text { in }\left(\mathrm{O}^{*}, \mathrm{I}\right), \Phi^{*}(\mathrm{I})=\pi\left(\frac{a_{0}}{2}+\frac{a_{1}}{2}\right), \Phi^{*}(\mu)=\pi\left(\begin{array}{c}
a_{0} \\
2
\end{array}+a_{1}\right) \text { in }(\mathrm{I}, 2), \\
\Phi^{*}(2)=\pi\left(\frac{a_{0}}{2}+a_{1}+\frac{a_{2}}{2}\right), \Phi^{*}(\mu)=\pi\left(\frac{a_{0}}{2}+a_{1}+a_{2}\right) \text { in }(2,3), \ldots, \\
\Phi^{*}(N)-\pi\left(\frac{a_{0}}{2}+a_{1}+\cdots+a_{N-1}+\frac{a_{N}}{2}\right), \Phi^{*}(\mu)=\pi\left(\frac{a_{0}}{2}+a_{1}+\cdots+a_{N}\right) \text { für } \mu>N .
\end{array}\right.
$$

Es ist also $\Phi^{*}(\mu)$ konstant in $(0,1),(1,2), \ldots(N-\mathrm{I}, N)$ und für $\mu>N$, und es ist:

$$
\begin{gathered}
\boldsymbol{\Phi}^{*}(+0)-\boldsymbol{\Phi}^{*}(0)=\pi \frac{a_{0}}{2}, \quad \boldsymbol{\Phi}^{*}(k+0)-\boldsymbol{\Phi}^{*}(k)=\pi{ }_{2}^{a_{k}}, \quad \boldsymbol{D}^{*}(k)-\boldsymbol{\Phi}^{*}(k-0)=\pi \frac{a_{k}}{2} \\
(k=\mathrm{I}, 2, \ldots, N) .
\end{gathered}
$$

Es handelt sich noch um die Untersuchung von $\Phi^{* *}(\mu)$. Für jedes Intervall $(\mathrm{o}<) \xi \leqq \mu \leqq \eta$ ist:

$$
\int_{\xi}^{\eta} h^{* *}(x) \stackrel{\sin \mu x}{x}-d x=\frac{1}{\xi} \int_{\xi}^{\xi *} h^{* *}(x) \sin \mu x d x
$$

Aus den Ungleichungen (48) folgt also: zu jedem $\varepsilon>0$ gibt es ein $b$, sodass für $b \leqq \xi<\eta$ und $o \leqq \mu \leqq N+\frac{\mathrm{I}}{2}$ :

$$
\left|\int_{j}^{\eta} h^{* *}(x) \stackrel{\sin \mu x}{x} d x\right|<\varepsilon ; \quad\left|\int_{-\eta}^{-\bar{j}} h^{* *}(x) \frac{\sin \mu x}{x} d x\right|<\varepsilon
$$

Es existiert also $\boldsymbol{\Phi}^{* *}(\mu)$ für $O \leqq \mu \leqq N+\frac{1}{2}$.

Wir gehen nun an die Berechnung von $\boldsymbol{\Phi}^{* *}(\mu)$, und wollen zeigen, dass sie aus:

$$
h^{* *}(x) \sim \sum_{k=N+1}^{\infty}\left(a_{k} \cos k x+b_{k} \sin k x\right)
$$

durch gliedweise Integration erfolgen kann.

Bekanntlich ist durch 


$$
H(x)=\sum_{k=N+1}^{\infty} \frac{\mathrm{I}}{k}\left(a_{k} \sin k x-b_{k} \cos k x\right)
$$

ein unbestimmtes Integral von $h^{* *}(x)$, durch:

$$
\bar{H}(x)=-\sum_{k-N+1}^{\infty} \frac{\mathrm{I}}{k^{2}}\left(a_{k} \cos k x+b_{k} \sin k x\right)
$$

ein unbestimmtes Integral von $H(x)$ gegeben, und die Reihen (52), (53) konvergieren gleichmässig (als Fouriersche Reihen totalstetiger Funktionen).

Durch zweimalige partielle Integration findet man:

$$
\begin{aligned}
& \left.\left.\int_{\xi}^{\eta} h^{* *}(x) \frac{\sin \mu x}{x} d x=H(x) \frac{\sin \mu x}{x}\right]_{\xi}^{\eta}+H(x)\left(\frac{\sin \mu x}{x^{2}}-\mu \frac{\cos \mu x}{x}\right)\right]_{\xi}^{\eta} \\
& +\int_{\dot{\zeta}}^{\eta} \bar{H}(x)\left(2 \frac{\sin \mu x}{x^{3}}-2 \mu \frac{\cos \mu x}{x^{2}}-\mu^{2^{\sin \mu x}} x\right) d x \\
& \left.=\sum_{k=N+1}^{\infty} \frac{1}{k}\left(a_{k} \sin k x-b_{k} \cos k x\right) \frac{\sin }{x} \frac{\mu x}{\eta}\right]_{j}^{\eta} \\
& \left.-\sum_{k=N+1}^{\infty} \frac{\mathrm{I}}{k^{2}}\left(a_{k} \cos k x+b_{k} \sin k x\right)\left(\frac{\sin \mu x}{x^{2}}-\mu^{\cos \mu x} \frac{\mu x}{x}\right)\right]_{\xi}^{\eta} \\
& -\sum_{k=N+1}^{\infty} \frac{1}{k^{2}} \int_{j}^{\eta}\left(a_{k} \cos k x+b_{k} \sin k x\right)\left(\begin{array}{c}
\sin \mu x \\
2-x^{3}-2 \mu \frac{\cos \mu x}{x^{2}}-\mu^{2} \sin \mu x \\
x
\end{array}\right) d x
\end{aligned}
$$

Aufgefasst als Funktionen von $\xi$ oder von $\eta$ sind die auf der rechten Seite auftretenden Reihen gleichmässig konvergent: die beiden ersten wegen der gleichmässigen Konvergenz der Reihen (52) und (53), die dritte, weil die darin auftretenden Integrale (für $\mathrm{O} \leqq \mu \leqq N+\frac{\mathrm{I}}{2}$ ) zwischen endlichen Schranken bleiben. Es kann also der Grenzübergang $\xi \rightarrow+\infty$ gliedweise vollzogen werden:

$$
\begin{aligned}
& \int_{-\infty}^{+\infty} h^{* *}(x) \frac{\sin \mu x}{x} d x=
\end{aligned}
$$

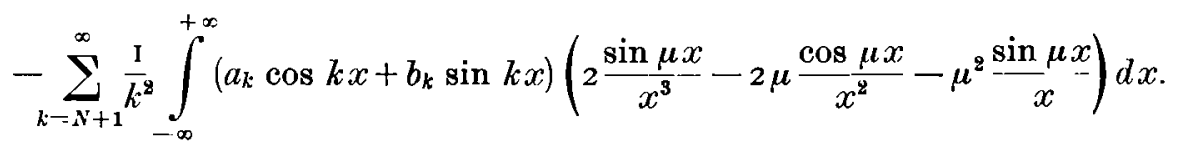


Wie zweimalige partielle Integration lehrt, ist aber $\left(\right.$ für $\left.k>N, \mathrm{o} \leqq \mu \leqq N+\frac{\mathrm{I}}{2}\right)$ :

$$
\begin{aligned}
& \int_{-\infty}^{+\infty}\left(a_{k} \cos k x+b_{k} \sin k x\right) \frac{\sin \mu x}{x} d x= \\
& \quad-\frac{1}{k^{2}} \int_{-\infty}^{+\infty}\left(a_{k} \cos k x+b_{k} \sin k x\right)\left(2-\frac{\sin \mu x}{x^{3}}-2 \mu \frac{\cos \mu x}{x^{2}}-\mu^{2} \frac{\sin \mu x}{x}\right) d x
\end{aligned}
$$

sodass wir schliesslich haben:

$$
\int_{-\infty}^{+\infty} h^{* *}(x)-\frac{\sin \mu x}{x} d x=\sum_{k-N+1}^{\infty} \int_{-\infty}^{+\infty}\left(a_{k} \cos k x+b_{k} \sin k x\right) \stackrel{\sin \mu x}{x} d x
$$

womit die Behauptung, dass $\boldsymbol{\Phi}^{* *}(\mu)$ durch gliedweise Integration berechnet werden kann, bewiesen ist.

Nach $\S 9$ ist aber für $k=N+\mathrm{I}, N+2, \ldots$ und $0 \leqq \mu \leqq N+\frac{\text { I }}{2}$ :

$$
\int_{-\infty}^{+\infty}\left(a_{k} \cos k x+b_{k} \sin k x\right) \frac{\sin \mu x}{x} d x=0
$$

es ist also:

$$
\boldsymbol{\Phi}^{* *}(\mu)=\text { o für } \mathrm{o} \leqq \mu \leqq N+\frac{\mathrm{I}}{2}
$$

Für diese Werte von $\mu$ stimmen also die Werte von $\boldsymbol{D}(\mu)$ mit den durch (5 I) gegebenen Werten von $\Phi^{*}(\mu)$ überein. Und da dabei $N$ ganz beliebig war, haben wir ganz allgemein:

$$
\begin{aligned}
& \Phi(o)=0, \Phi(\mu)=\pi \frac{a_{0}}{2} \text { für } \circ<\mu<\mathrm{I}, \boldsymbol{\Phi}(\mu)=\pi\left(\frac{a_{0}}{2}+a_{1}+\cdots+a_{k}\right) \text { für } k<\mu<k+\mathrm{I} \\
& (k=\mathbf{1}, 2, \ldots) \\
& D(k)=-\pi\left(\frac{a_{0}}{2}+a_{1}+\cdots+a_{k-1}+\frac{a_{k}}{2}\right) \\
& (k=\mathrm{I}, 2, \ldots) \text {. }
\end{aligned}
$$

Ganz ebenso findet man (abgesehen von einer additiven Konstante, auf die es nicht ankommt): 
Über eine Verallgemeinerung der Fourierschen Integralformel.

$\Psi(\mu)=$ o für $\mathrm{o} \leqq \mu<\mathrm{I} ; \Psi(\mu)=\pi\left(b_{1}+b_{\mathbf{g}}+\cdots+b_{k}\right)$ für $k<\mu<k+\mathrm{I} \quad(k=\mathrm{I}, 2, \ldots)$

$\Psi(\mathrm{I})=\pi_{2}^{b_{1}}, \Psi(k)=\pi\left(b_{1}+\cdots+b_{k-1}+\begin{array}{c}b_{k} \\ 2\end{array}\right)$

$(k=\mathrm{I}, 2, \ldots)$

Infolgedessen ist:

$\int_{0}^{\lambda}\left(\cos \mu x_{0} d \Phi(\mu)+\sin \mu x_{0} d \Psi(\mu)\right)=$
$=\pi\left(\frac{a_{0}}{2}+\sum_{k=1}^{n}\left(a_{k} \cos k x_{0}+b_{k} \sin k x_{0}\right)\right)$ für $n<\lambda<n+\mathrm{I}$,
$\int_{0}^{n}\left(\cos \mu x_{0} d \Phi(\mu)+\sin \mu x_{0} d \Psi(\mu)\right)=$
$=\pi\left(\begin{array}{c}a_{0} \\ 2\end{array}+\sum_{k=1}^{n-1}\left(a_{k} \cos k x_{0}+b_{k} \sin k x_{0}\right)+{ }_{2}^{\mathrm{I}}\left(a_{n} \cos n x_{0}+b_{n} \sin n x_{0}\right)\right)$.

Hat nun $f(x)$ die Eigenschaft $\mathbf{A})$, so gilt:

$$
f\left(x_{0}\right)=\frac{a_{0}}{2}+\sum_{k=1}^{\infty}\left(a_{k} \cos k x_{0}+b_{k} \sin k x_{0}\right),
$$

und wir erhalten somit:

Satz IX. Ist $f(x)$ eine periodische Funktion der Figenschaft A), so gilt Formel (10); und zwar reduziert sich dann die rechte Seite von (10) auf dic Fouriersche Reihenentwicklung von $f\left(x_{0}\right)$.

Wir sehen also, dass unsere Formel (Io) die sämtlichen konvergenten Fourierschen Reihen umfasst.

$\S$ I6.

Nun behandeln wir noch den Fall:

$$
f(x)==f_{0}(x) h(x)
$$

wo $f_{0}(x)$ die Funktion (3I) bedeutet, und $h(x)$ periodisch ist. Wieder können wir annehmen, $h(x)$ habe die Periode $2 \pi$. Wir stellen dieselben Utberlegungen wie in $\$$ I 4 an. 
Aus $\$ \$ 8$, Io und I I entnehmen wir: $\boldsymbol{\Phi}^{*}(\mu)$ ist stetig für $\mu \geqq 0$ und $\neq \mathrm{I}, 2, \ldots, N$, und es ist:

$$
\lim _{h \rightarrow 0}\left(\boldsymbol{\Phi}^{*}(k+h)-\boldsymbol{\Phi}^{*}(k-h)\right)=0 \quad(k=1,2, \ldots, N)
$$

$\Psi^{*}(\mu)$ ist stetig für $\mu>0$ und $\neq \mathrm{I}, 2, \ldots, N$, und es ist:

$$
\lim _{h \rightarrow 0}\left(\Psi^{*}(k+h)-\Psi^{*}(k-h)\right)=0 \quad(k=\mathrm{I}, 2, \ldots, N)
$$

Wie in $\S$ I4 sehen wir: $\Phi^{* *}(\mu)$ und $\Psi^{* *}(\mu)$ existieren und sind stetig für $0 \leqq \mu<$ $<N+$ I. Daraus folgt (da $N$ beliebig ist): $\boldsymbol{\Phi}(\mu)$ existiert und ist stetig für $\mu \geqq 0$ und $\neq 1,2, \ldots ; \Psi(\mu)$ existiert und ist stetig für $\mu>0$ und $\neq 1,2, \ldots$, und es ist:

$$
\lim _{h \rightarrow 0}(\boldsymbol{\Phi}(k+h)-\boldsymbol{\Phi}(k-h))=\mathrm{o}, \quad \lim _{l \rightarrow 0}(\Psi(k+h)-\Psi(k-h))=0 \quad(k=\mathrm{I}, 2, \ldots) .
$$

Da auch alles über das Integral $I(\lambda)$ in $\$ 14$ Bewiesene hier giltig bleibt, erhalten wir:

Satz X. Die Aussage von Satz VIII gilt auch fï jede Funktion der Eigenschaft A), die die Gestalt hat: $f(x)=f_{0}(x) h(x)$, wo $f_{0}(x)$ die Funktion (3r) bedeutet, und $h(x)$ die Periode $p$ hat.

Die Utberlegungen von $\S 8$ gestatten es nun, die Sätze VIII, IX, X zusammenzufassen. Sei $f(x)=g(x) h(x)$, wo $g(x)$ im Unendlichen beschränkt und monoton, $h(x)$ periodisch ist. Wie in $\$ 8$ setzen wir:

$$
g(x)==\frac{c_{1}+c_{2}}{2}+\frac{c_{1}-c_{2}}{2} f_{0}(x)+g_{1}(x)
$$

wo:

$$
c_{1}=\lim _{x \rightarrow+\infty} g(x), \quad c_{2}=\lim _{x \rightarrow+\infty} g(x) .
$$

Dann konvergiert $g_{1}(x)$ im Unendlichen monoton gegen o. Wir finden, wenn zunächst wieder $h(x)$ die Periode $2 \pi$ und die Fourierreihe (46) hat: Die zu $f(x)$ gehörige Funktion $\Phi(\mu)$ existiert für $\mu \geqq 0$ und ist stetig für $\mu=\vDash 0,1,2, \ldots$; die Funktion $\Psi(\mu)$ existiert und ist stetig für $\mu>0$ und $\neq \mathrm{I}, 2, \ldots$. Hs ist

$$
\begin{gathered}
\boldsymbol{\Phi}(+\mathrm{o})-\boldsymbol{\Phi}(\mathrm{o})=\pi \cdot \frac{c_{1}+c_{2}}{2} \cdot \frac{a_{0}}{2} \lim _{h \rightarrow+0}(\boldsymbol{\Phi}(k+h)-\boldsymbol{\Phi}(k-h))=\pi \cdot \frac{c_{1}+c_{9}}{2} \cdot a_{k} \quad(k=\mathrm{I}, 2, \ldots) \\
\lim _{h \rightarrow+0}(\Psi(k+h)-\Psi(k-h))=\pi \cdot \frac{c_{1}+c_{9}}{2} b_{k}
\end{gathered}
$$

und es gilt: 
Über eine Verallgemeinerung der Fourierschen Integralformel.

Satz XI. Ist $f(x)$ eine Funktion der Eigenschaft A), die die Gestalt hat: $f(x)=g(x) h(x)$, wo $g(x)$ im Unendlichen beschränkt und monoton ist und $h(x)$ die Periode $p$ hat, so gilt Formel (10). Dabei ist in ihr $\int_{0}^{+\infty}=\lim _{v \rightarrow \infty} \int_{0}^{\lambda_{v}} z u$ setzen, wo $\lambda_{v}$ eine beliebige Folge positiver Zahlen mit $\lambda_{\nu} \rightarrow+\infty$ bedeutet, deren Glieder keinem der Intervalle $\left|\lambda-\frac{2 k \pi}{p}\right|<\delta(k=\mathbf{I}, 2, \ldots)$ angehören ( $\delta$ eine beliebige positive Zahl), und unter $\int_{0}^{\hat{\lambda}}$, wenn $\lambda$ zwischen $\frac{2 n \pi}{p}$ und $\frac{2(n+\mathrm{I}) \pi}{p}$ liegt, der Grenzuert zu verstehen ist:

$$
\begin{aligned}
& \int_{0}^{\lambda}=\lim _{h_{0} \rightarrow+0, \ldots, h_{n} \rightarrow+0}\left(\sum_{k=1}^{n} \int_{\frac{2(k \cdots-1) \pi}{p}+h_{k-1}}^{2 k \pi}+\int_{\frac{2 n \pi}{p}+h_{n}}^{\lambda}\right) \\
& +\lim _{h \rightarrow+0}(\boldsymbol{\Phi}(h)-\boldsymbol{\Phi}(\mathrm{o}))+\sum_{k=1}^{n}\left\{\cos \frac{2 k \pi}{p} x_{0} \lim _{h \rightarrow+0}\left(\boldsymbol{\Phi}\left(\frac{2 k \pi}{p}+h\right)-\boldsymbol{\Phi}\left(\frac{2 k \pi}{p}-h\right)\right)\right. \\
& \left.+\sin \frac{2 k \pi}{p} x_{0} \lim _{h \rightarrow+0}\left(\Psi\left(\frac{2 k \pi}{p}+h\right)-\Psi\left(\begin{array}{c}
2 k \pi \\
p
\end{array}-h\right)\right)\right\} .
\end{aligned}
$$

Nun wollen wir annehmen, $f(x)$ habe die Gestalt:

$$
f(x)==\sum_{k=1}^{\infty}\left(a_{k} \cos q_{k} x+b_{k} \sin q_{k} x\right)
$$

wo alle $q_{k}>0$, und die Reihe

$$
\sum_{k=1}^{\infty}\left(\left|a_{k}\right|+\left|b_{k}\right|\right)
$$

konvergent sei.

Wir zerlegen $f(x)$ in $f_{1}(x)+f_{2}(x)$, wo

$$
f_{1}(x)=\sum_{k=1}^{\infty} a_{k} \cos q_{k} x ; \quad f_{2}(x)=\sum_{k=1}^{\infty} b_{k} \sin q_{k} x .
$$

44-2661. Ach mathematica. 49. Imprimé It 31 juillet 1926. 
Entsprechend zerlegt sich $\Phi(\mu)$ in $\Phi_{1}(\mu)+\Phi_{2}(\mu)$; da aber $f_{8}(x)$ ungerade, ist $\Phi_{2}(\mu)=$ o, mithin $\Phi(\mu)=\Phi_{1}(\mu)$. Da die Reihe für $f_{1}(x)$ gleichmässig konvergiert, ist für jedes endliche Intervall $[\xi, \eta]$ :

$$
\int_{\xi}^{\eta} f_{1}(x) \stackrel{\sin \mu x}{x} d x=\sum_{k=1}^{\infty} a_{k} \int_{\xi}^{\eta} \cos q_{k} x \frac{\sin \mu x}{x} d x,
$$

wo die rechts stehende Reihe wieder gleichmässig für alle $\xi$ und $\eta$ konvergiert, so dass die Grenzübergänge $\xi \rightarrow-\infty, \eta \rightarrow+\infty$ gliedweise vollzogen werden können. So erhalten wir:

$$
\boldsymbol{\Phi}(\mu)=\int_{-\infty}^{+\infty} f_{1}(x)^{\sin \mu x} x d x=-\sum_{k=1}^{\infty} a_{k} \int_{-\infty}^{+\infty} \cos q_{k} x \stackrel{\sin \mu x}{x} d x
$$

wo die rechts stehende Reihe wieder gleichmässig in $\mu$ konvergiert.

Aufgrund der Resultate von $\S 8$ und 9 ersehen wir daraus: die Funktion $\Phi(\mu)$ ist (für $\mu \geqq 0)$ konstant in jedem Intervalle, das keinen Punkt $q_{k}(k=\mathrm{I}, 2 \ldots)$ enthält; sie ist stetig in jedem von allen $q_{k}$ verschiedenem Punkte, während sie in den Punkten $q_{k}$ unstetig ist gemäss:

$$
\boldsymbol{\Phi}\left(q_{k}+0\right)-\boldsymbol{\Phi}\left(q_{k}\right)=\frac{\pi}{2} a_{k} ; \quad \boldsymbol{\Phi}\left(q_{k}\right)-\boldsymbol{\Phi}\left(q_{k}-\mathrm{o}\right)=\frac{\pi}{2} a_{k} \quad(k=\mathrm{I}, 2, \ldots) .
$$

Ebenso findet man: die Funktion $\Psi(\mu)$ ist (für $\mu \geqq 0$ ) konstant in jedem Intervalle, das keinen Punkt $q_{k}(k=\mathrm{I}, 2, \ldots)$ enthält, sie ist stetig in jedem von allen $q_{k}(k=\mathrm{I}, 2, \ldots)$ verschiedenem Punkte, während sie in den Punkten $q_{k}$ unstetig ist gemäss:

$$
\Psi\left(q_{k}+0\right)-\Psi\left(q_{k}\right)=\frac{\pi}{2} b_{k} ; \quad \Psi\left(q_{k}\right)-\Psi\left(q_{k}-0\right)=\frac{\pi}{2} b_{k}
$$

Daraus folgt unmittelbar:

Satz XII. Ist $f(x)$ gegeben durch (54), wo die Reihe (55) konvergiert, so gilt Formel (1о); und zwar reduziert sich die rechte Seite von (го) auf die Reihe (54) fiir $x=x_{0}$. 


\section{$\S 18$.}

Nunmehr nehmen wir an, $f(x)$ habe die Gestalt:

$$
f(x)=g(x) h(x),
$$

wo $g(x)$ im Unendlichen beschränkt und monoton sei, und $h(x)$ gegeben sei durch:

$$
h(x)=\sum_{k=1}^{\infty}\left(a_{k} \cos q_{k} x+b_{k} \sin q_{k} x\right)
$$

wo die $q_{k}$ eine monoton ins Unendlichen wachsende Folge positiver Zahlen bedeuten, und wieder die Reihe (55) konvergiere.

Wir nehmen mit $g(x)$ wieder die Zerlegung (32) vor:

$$
g(x)=\frac{c_{1}+c_{2}}{2}+\frac{c_{1}-c_{2}}{2} f_{0}(x)+g_{1}(x),
$$

und können uns, da wir über den Beitrag des Summanden $\frac{c_{1}+c_{2}}{2}$ durch $§$ I 7 völlig informiert sind, auf die Betrachtung der beiden anderen Summanden beschränken. Es bedeute also im Folgenden $g(x)$, entweder die Funktion $f_{0}^{\prime}(x)$, oder eine Funktion, die im Unendlichen monoton gegen o konvergiert. In beiden Fällen konvergiert $\frac{g(x)}{x}$ im Unendlichen monoton gegen 0 .

Ist $g(x)$ monoton für $x>a$, so gilt für $a<\xi<\eta$ :

$$
\begin{aligned}
\int_{\xi}^{\eta} f(x) \frac{\sin }{x} \frac{\mu x}{x} d x=\frac{g(\xi)}{\xi} \int_{\xi}^{\xi *} h(x) \sin \mu x d x \\
=\frac{g(\xi)}{\xi} \sum_{k-1}^{\infty}\left(a_{k} \int_{\xi}^{\xi} \cos q_{k} x \sin \mu x d x+b_{k} \int_{\xi}^{\xi} \sin q_{k} x \sin \mu x d x\right) .
\end{aligned}
$$

Nun gehört zu jedem $\delta>0$ ein $M$, so dass, wenn $\mu \geqq 0$ allen Ungleichungen genügt $\left|\mu-q_{k}\right| \geqq \delta(k=\mathrm{I}, 2, \ldots)$, für alle $\xi$ und $\xi^{*}$ die Ungleichungen bestehen:

$$
\left|\int_{\xi}^{\xi} \cos q_{k} x \sin \mu x d x\right|<M, \quad\left|\int_{\xi}^{*} \sin q_{k} x \sin \mu x d x\right|<M,
$$


und mithin für alle $\xi$ und $\eta$ :

$$
\left|\int_{\xi}^{\eta} f(x) \frac{\sin \mu x}{x} d x\right|<\left|\frac{g(\xi)}{\xi}\right| \cdot M \cdot \sum_{k=1}^{\infty}\left(\left|a_{k}\right|+\left|b_{k}\right|\right)
$$

Es gibt also zu jedem $\varepsilon>0$ und $\delta>0$ ein $b$, sodass für $b \leqq \xi<\eta$ und alle $\mu \geqq 0$, die den sämtlichen Ungleichungen $\left|\mu-q_{k}\right| \geqq \delta(k==\mathrm{I}, 2, \ldots)$ genügen, die Ungleichung gilt:

$$
\left|\int_{\xi}^{\eta} f(x) \frac{\sin \frac{\mu x}{x}}{x} d x\right|<\varepsilon
$$

Wie immer entnehmen wir hieraus: Für alle $\mu \geqq 0$ und $\neq q_{k}$ existiert $\Phi(\mu)$ und ist stetig. In jedem Intervalle $(o \leqq) \alpha \leqq \mu \leqq \beta$, das keinen der Punkte $q_{k}$ enthält, konvergiert die durch (27) definierte Funktion $\Phi_{n}(\mu)$ gleichmässig gegen $\Phi(\mu)$. Und die analogen Aussagen gelten für $\Psi(\mu)$.

Um das Verhalten von $\boldsymbol{\Phi}(\boldsymbol{\mu})$ und $\Psi(\mu)$ an der Stelle $q_{k}$ festzustellen, setzen wir:

$$
h^{*}(x)=h(x)-\left(a_{k} \cos q_{k} x+b_{k} \sin q_{k} x\right) .
$$

Die zu $f^{*}(x)=g(x) h^{*}(x)$ gehörige Funktion $\Phi$ bleibt dann nach dem eben Bewiesenen für $\mu=q_{k}$ stetig; es verhält sich also die zu $f(x)$ gehörige Funktion $\boldsymbol{\Phi}(\mu)$ für $\mu=q_{k}$ so wie $\int_{-\infty}^{+\infty} g(x)\left(a_{k} \cos q_{k} x+b_{k} \sin q_{k} x\right) \frac{\sin \mu x}{x} d x$, woraus nach $\S$ Io, I I, I 2 sofort folgt:

$$
\lim _{h \rightarrow 0}\left(\boldsymbol{\Phi}\left(q_{k}+h\right)-\boldsymbol{\Phi}\left(q_{k}-h\right)\right)=0
$$

und ebenso beweist man:

$$
\lim _{h \rightarrow 0}\left(\Psi\left(q_{k}+h\right)-\Psi\left(q_{k}-h\right)\right)=\mathrm{o}
$$

In gewohnter Weise folgern wir aus diesen Resultaten, dass das Integral:

$$
I(\lambda)=\int_{-\infty}^{+\infty} f(x) \frac{\sin \lambda\left(x-x_{0}\right)}{x-x_{0}} d x
$$

für alle $\lambda \geqq 0$ und $\neq q_{k}(k=\mathrm{I}, 2, \ldots)$ existiert und stetig ist, dass: 


$$
\lim _{h \rightarrow 0}\left(I\left(q_{k}+h\right)-I\left(q_{k}-h\right)\right)=0
$$

ist, dass falls $f(x)$ die Eigenschaft $\mathbf{A})$ hat, für jede Folge positiver Zahlen $\lambda_{v}$ mit $\lambda_{v} \rightarrow+\infty$; die keinem der Intervalle $\left|\lambda-q_{k}\right|<\delta(k=\mathrm{I}, 2, \ldots)$ angehören:

$$
f\left(x_{0}\right)=\frac{\mathrm{I}}{\pi} \lim _{\nu \rightarrow \infty} I\left(\lambda_{\nu}\right)
$$

ist, und dass für jedes Intervall $(\mathrm{o} \leqq) \alpha \leqq \mu \leqq \beta$, das keinen der Punkte $q_{k}$ enthält:

$$
I(\beta)-I(\alpha)=\int_{\alpha}^{\beta}\left(\cos \mu x_{0} d \Phi(\mu)+\sin \mu x_{0} d \Psi(\mu)\right)
$$

ist. Daraus ergibt sich:

Satz XIII. Ist $f^{\prime}(x)=g(x) . h(x)$, wo $g(x)$ entweder die durch (3 I) definierte Funktion $f_{0}(x)$ bedeutet, oder im Unendlichen monoton gegen o konvergiert, und $h(x)$ die Bedeutung (56) mit konvergenter Reihe (55) hat, so gilt, falls $f(x)$ die Eigenschaft A) hat, und $\delta>0$ so klein gewählt werden kann, dass es eine Folge positiver. Zahlen $\lambda_{v}$ mit $\lambda_{v} \rightarrow+\infty$ gibt, die keinem der. Intervalle $\left|\lambda_{v}-g_{k}\right|<\delta(k=\mathrm{I}, 2, \ldots)$ angehören, Formel (Io). Es ist in ihr $\int_{0}^{+\infty}=\lim _{v \rightarrow \infty} \int_{0}^{\lambda_{y}} z u$ setzen und für $\lambda=q_{k}(k=\mathrm{I}, 2, \ldots)$ der Cauchysche Hauptwert zu nehmen.

Durch Kombination von Satz XII und XIII erhalten wir:

Satz XIV. Ist $f(x)=g(x) h(x)$, wo $g(x)$ im Unendlichen beschränkt und monoton ist, und $h(x)$ die Bedeutung (56) mit konvergenter Reihe (55) hat, so gilt, falls $f(x)$ die Figenschaft A) hat, und $\delta>0$ so klein gewählt werden kann, dass es eine Folge positiver Zahlen $\lambda_{\nu}$ mit $\lambda_{\nu} \rightarrow+\infty$ gibt, die keinem der Intervalle $\left|\lambda-q_{k}\right|<\delta$ $(k=\mathrm{I}, 2, \ldots)$ angehören, Formel (10). Es ist in ihr $\int_{0}^{+\infty}=\lim _{\boldsymbol{v} \rightarrow \infty} \int_{0}^{\lambda_{v}} z u$ setzen und unter $\int_{0}^{\lambda}$, wenn $q_{n}<\lambda<q_{n+1}$ ist, der Grenzwert zu verstehen: 


$$
\begin{aligned}
\int_{0}^{\lambda}= & \lim _{l_{1} \rightarrow+0, \ldots, h_{n} \rightarrow+0}\left(\int_{0}^{q_{1}-h_{1}}+\sum_{k=1}^{n-1} \int_{q_{k}+h_{k}}^{q_{k+1}-h_{k+1}}+\int_{q_{n}+h_{n}}^{\lambda}\right) \\
& +\sum_{k=1}^{n}\left\{\cos q_{k} x_{0} \lim _{l \rightarrow+0}\left(\Phi\left(q_{k}+h\right)-\Phi\left(q_{k}-h\right)\right)+\sin q_{k} x_{0} \lim _{h \rightarrow+0}\left(\Psi\left(q_{k}+h\right)-\Psi\left(q_{k}-h\right)\right)\right\} .
\end{aligned}
$$

Zum Schlusse betrachten wir noch Funktionen $f(x)$, denen folgende Eigenschaft zukommi:

D) Es sei $f(x)$ im Unendlichen quadratisch integrierbar (d. h. es gebe ein $a$, so dass die beiden Grenzwerte $\lim _{q \rightarrow+\infty} \int_{a}^{q}(f(x))^{2} d x$ und $\lim _{q \rightarrow+\infty} \int_{-q}^{-a}(f(x))^{2} d x$ endlich ausfallen).

Wir erkennen sofort: hat $f(x)$ die Eigenschaften A) und D), so gilt Formel (I). In der Tat, die Schwarzsche Ungleichung ergibt für jedes Intervall $[\xi, \eta]$ :

$$
\begin{array}{r}
\left|\int_{\xi}^{\eta} f(x) \frac{\sin \lambda\left(x-x_{0}\right)}{x-x_{0}} d x\right| \leqq\left(\int_{\xi}^{\eta}(f(x))^{2} d x\right)^{1} \cdot\left(\int_{\xi}^{\eta}\left(\begin{array}{c}
\sin \lambda\left(x-x_{0}\right) \\
x-x_{0}
\end{array}\right)^{2} d x\right)^{\frac{1}{2}} \\
<M \cdot\left(\int_{\xi}^{\eta}(f(x))^{2} d x\right)^{\frac{1}{2}}
\end{array}
$$

wo $M$ eine geeignete Konstante bedeutet. Daraus folgt: zu jedem $\varepsilon>0$ gibt es ein $b$, so dass für $b \leqq \xi<\eta$ :

$$
\left|\int_{\xi}^{\eta} f(x) \frac{\sin \lambda\left(x-x_{0}\right)}{x-x_{0}} d x\right|<\varepsilon, \quad\left|\int_{-\eta}^{-\xi} f(x)^{\sin \lambda\left(x-x_{0}\right)} x\right|<\varepsilon
$$

woraus in bekannter Weise die Behauptung folgt.

Wir definieren wieder $\boldsymbol{\Phi}(\mu)$ und $\Psi(\mu)$ durch $(7)$. Genau wie eben ergibt die Schwarzsche Ungleichung, dass $\Phi(\mu)$ und $\Psi(\mu)$ für alle $\mu \geqq 0$ existieren, und dass auch hier die Beziehungen (8) gleichmässig erfüllt sind. Daraus folgt, dass 
$\Phi(\mu)$ und $\Psi(\mu)$ für alle $\mu \geqq 0$ stetig sind, und ganz wie in § I gelangt man zum Satze:

Satz XV. Fïr jede Funktion der Eigenschaften A) und D) gilt Formel (10).

Sei $\mu_{1}^{\prime}, \mu_{2}^{\prime}, \ldots, \mu_{n}^{\prime}, \ldots$ eine monotone Folge negativer Zahlen mit $\mu_{n}^{\prime} \rightarrow-\infty$, und $\mu_{1}^{\prime \prime}, \mu_{2}^{\prime \prime}, \ldots, \mu_{n}^{\prime \prime}, \ldots$ eine monotone Folge positiver Zahlen mit $\mu_{n}^{\prime \prime} \rightarrow+\infty$. Wir setzen:

$$
\Phi^{(n)}(\mu)=\int_{\mu_{n}^{\prime}}^{\mu_{n}^{\prime \prime}} f(x) \frac{\sin \mu x}{x} d x ; \quad \Psi(n)(\mu)=\int_{\mu_{n}^{\prime}}^{\mu_{n}^{\prime \prime}} f(x) \frac{\mathrm{I}-\cos \mu x}{x} d x
$$

$$
\varphi^{(n)}(\mu):=\int_{\mu_{n}^{\prime}}^{\mu_{n}^{\prime \prime}} f(x) \cos \mu x d x ; \quad \psi^{(n)}(\mu)=\int_{\mu_{n}^{\prime}}^{\mu_{n}^{\prime \prime}} f(x) \sin \mu x d x
$$

dann ist:

$$
\Phi^{(n)}(\mu)=\int_{0}^{\mu} \varphi^{(n)}(\mu) d \mu, \quad \Psi^{(n)}(\mu)==\int_{0}^{\mu} \psi^{(n)}(\mu) d \mu
$$

Wir zeigen: die Folge der $\varphi^{(n)}(\mu)$, sowie die Folge der $\psi^{(n)}(\mu)$ ist im Intervalle $(\mathrm{o},+\infty)$ im Mittel konvergent.

In der Tat, nach dem Parsevalschen Theorem für Fouriersche Integrale ist:

$$
\frac{\mathrm{I}}{\pi} \int_{0}^{+\infty}\left\{\left(\varphi^{\left(n_{1}\right)}(\mu)-\varphi^{\left(n_{3}\right)}(\mu)\right)^{2}+\left(\psi^{\left(n_{1}\right)}(\mu)-\psi^{\left(n_{z}\right)}(\mu)\right)^{2}\right\} d \mu=\int_{\mu_{n_{3}}^{\prime}}^{\mu_{n_{1}}^{\prime}}(f(x))^{2} d x+\int_{\mu_{n_{1}}^{\prime \prime}}^{\mu_{n_{2}}^{\prime \prime}}(f(x))^{2} d x
$$

Aus Eigenschaft D) von $f(x)$ ergibt sich hieraus unmittelbar: zu jedem $\varepsilon>0$ gibt es ein $N$, so dass für $n_{1} \geqq N, n_{2} \geqq N$ :

$$
\int_{0}^{+\infty}\left(\varphi^{\left(n_{1}\right)}(\mu)-\varphi^{\left(n_{2}\right)}(\mu)\right)^{2} d \mu<\varepsilon, \quad \int_{0}^{+\infty}\left(\psi^{\left(n_{1}\right)}(\mu)-\psi^{\left(n_{2}\right)}(\mu)\right)^{2} d \mu<\varepsilon
$$

womit die Behauptung bewiesen ist. 
Fs konvergieren also die $\varphi^{(n)}(\mu)$ im Mittel gegen eine Funktion $\varphi^{*}(\mu)$, die $\psi^{(n)}(\mu)$ gegen eine Funktion $\psi^{*}(\mu)$. Bekanntlich folgt daraus für alle $\mu \geqq 0$ :

$$
\lim _{n \rightarrow \infty} \int_{0}^{\mu} \varphi^{(n)}(\mu) d \mu=\int_{0}^{\mu} \varphi^{*}(\mu) d \mu ; \quad \lim _{n \rightarrow \infty} \int_{0}^{\mu} \psi^{(n)}(\mu) d \mu=\int_{0}^{\mu} \psi^{*}(\mu) d \mu .
$$

Wegen (58) und (59) kann das geschrieben werden:

$$
\Phi(\mu)=\int_{0}^{\mu} \varphi^{*}(\mu) d \mu, \quad \Psi(\mu)=\int_{0}^{\mu} \psi^{*}(\mu) d \mu
$$

und durch Einsetzen in (10) erhalten wir:

Satz XVI. Für jede Funktion $f(x)$ der Eigenschaften A) und D) gilt:

$$
f\left(x_{0}\right)=\frac{\mathrm{I}}{\pi} \int_{0}^{+\infty}\left(\varphi^{*}(\mu) \cos \mu x_{0}+\psi^{*}(\mu) \sin \mu x_{0}\right) d \mu
$$

dabei bedeuten $\varphi^{*}(\mu)$ und $\psi^{*}(\mu)$ die Funktionen, gegen die die durch $(57)$ definierten Funktionen $\varphi^{(n)}(\mu)$ bzw. $\psi^{(n)}(\mu)$ im Intervalle $(\mathrm{o},+\infty)$ im Mittel konvergieren, uenn $\mu_{n}^{\prime} \rightarrow-\infty, \mu_{n}^{\prime \prime} \rightarrow+\infty$.

Der Unterschied gegenüber der Fourierschen Integralformel (I I) ist der, dass wir nicht behaupten können, dass $\varphi^{*}(\mu)$ und $\psi^{*}(\mu)$ durch die Integrale (I2) gegeben sind, da diese letzteren nicht zu existieren brauchen. Doch ergeben bekannte Sätze:

Satz XVII. Für jede Funktion $f(x)$ der Eigenschaften $A$ ) und D) gilt die Fouriersche Integralformel (I I), falls die durch (I 2) definierten Funktionen $\varphi(\mu)$ und $\psi(\mu)$ abgesehen von einer Nullmenge existieren.

In der Tat, da die $\varphi^{(n)}(\mu)$ im Mittel gegen $\varphi^{*}(\mu)$ konvergieren, kann aus ihnen eine Teilfolge $\varphi^{\left(n_{i}\right)}(\mu)$ herausgegriffen werden, die für alle $\mu \geqq 0$, abgesehen von einer Nullmenge, gegen $\varphi^{*}(\mu)$ konvergiert:

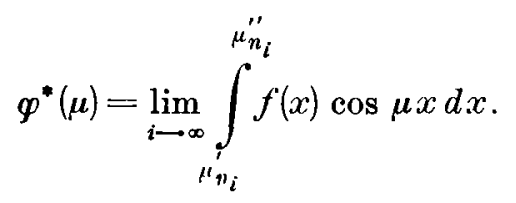


Wenn aber $\varphi(\mu)$ existiert, stimmt dieser Grenzwert mit $\varphi(\mu)$ überein; und dasselbe gilt für $\psi^{*}(\mu)$ und $\psi(\mu)$. Also folgt Satz XVII aus Satz XVI.

Nach einem Satze von M. Pranchured ${ }^{1}$ existieren $\varphi(\mu)$ und $\psi(\mu)$, abgesehen von einer Nullmenge, sicher dann, wenn das Integral $\int_{0}^{+\infty}(f(x))^{2}(\log x)^{2} d x$ existiert.

1 Math. Ann. 76 (I915), S. 315-326. 